\title{
ERGODIC OPTIMIZATION FOR HYPERBOLIC FLOWS AND LORENZ ATTRACTORS
}

\author{
MARCUS MORRO, ROBERTO SANT'ANNA AND PAULO VARANDAS
}

\begin{abstract}
In this article we consider the ergodic optimization for hyperbolic flows and Lorenz attractors with respect to both continuous and Hölder continuous observables. In the context of hyperbolic flows we prove that a Baire generic subset of continuous observables have a unique maximizing measure, with full support and zero entropy, and that a Baire generic subset of Hölder continuous observables admit a unique and periodic maximizing measure. These results rely on a relation between ergodic optimization for suspension semiflows and ergodic optimization for the Poincare map with respect to induced observables, which allow us to reduce the problem for the context of maps. Using that singular-hyperbolic attractors are approximated by hyperbolic sets, we obtain related results for geometric Lorenz attractors.
\end{abstract}

\section{INTRODUCTION AND STATEMENT OF THE MAIN RESULTS}

Let $X$ be a compact metric space, $f: X \rightarrow X$ be a continuous map and $\mathcal{M}_{f}$ be the collection of $f$-invariant Borel probability measures on $X$. The objects of interest in the field of ergodic optimization are those $f$ invariant probability measures which maximize, or minimize, the space average $\int \varphi d \mu$, for $\varphi: X \rightarrow \mathbb{R}$, over all $\mu \in \mathcal{M}_{f}$. These are the maximizing measures, or minimizing, measures for the function $\varphi$ (with respect to the dynamical system $f$ ). As usual, we restrict our attention to maximizing measures, since a minimizing measure for $\varphi$ is a maximizing measure for $-\varphi$. The compactness of $\mathcal{M}_{f}$ and continuity of the function $\mu \mapsto \int \varphi d \mu$ ensures that maximizing measures always exist. It is also clear from the ergodic decomposition theorem that almost all ergodic components of a maximizing measure are maximizing measures, hence ergodic maximizing measures also exist. Hence, some of the fundamental question in ergodic optimization are:

- What can we say about the maximizing measures?

$\circ$ Is there only one maximizing measure for typical observables?

- Can we describe the support of a maximizing measure?

$\circ$ Are maximizing measures typically periodic?

There exists an extensive list of contributions to these problems built over different approaches, some of which inspired by statistical mechanics and thermodynamic formalism (zero temperature limits) and others from the theory of cohomology equations (construction of sub-actions). In the known situations, the answer to the previous questions usually depend on the class of the dynamics but also on the regularity of the observables. In [31] Mañé conjectured that for a generic Lagrangian there exists a unique minimizing measure, and it is supported by a periodic orbit. Contreras, Lopes and Thieullen [17] and later Contreras [18] obtained a proof of this conjecture in the case of expanding maps. For an account on the many contributions to this problem we refer the reader to $[5,9,10,11,16,20,21,24,25,33,34,37,44]$ and references therein. Based on various approaches utilized in the literature, we can emphasize that the regularity of the observables plays an important role on the proofs: for Lipschitz potentials, one can obtain maximizing measures supported in periodic orbits, whereas for continuous potentials, the support of the maximizing measure is the whole space. We refer the reader to $[4,26,27]$ for excellent surveys on ergodic optimization.

2000 Mathematics Subject Classification. primary 37D20, 37C10, 37C50, secondary 37C27. 37A05, Key words and phrases. Ergodic optimization, hyperbolic flows, gluing orbit property, Lorenz attractors. 
Here we will address on the ergodic optimization for hyperbolic and singular-hyperbolic flows with respect to both continuous and Hölder continuous observables. Let $M$ be a closed Riemannian manifold and $\left(X^{t}\right)_{t}: M \rightarrow$ $M$ a smooth flow. Given a continuous function $\varphi: M \rightarrow \mathbb{R}$ a maximizing measure for $\left(X^{t}\right)_{t}$ with respect to $\varphi$ is a $\left(X^{t}\right)_{t}$-invariant Borel probability measure $\mu$ so that

$$
\int \varphi d \mu=\max \left\{\int \varphi d v: v \in \mathcal{M}_{1}\left(M,\left(X^{t}\right)_{t}\right)\right\} .
$$

Maximizing measures always exist because $\mathcal{M}_{1}\left(M,\left(X^{t}\right)_{t}\right)$ is compact in the weak* topology and $v \mapsto \int \varphi d \mu$ is continuous. First results on the ergodic optimization for flows were due to Lopes and Thieullen [29] and Pollicott and Sharp [36] where the authors constructed sub-actions for Anosov flows (related results include [30] in the context of expansive geodesic flows). The construction of calibrated sub-actions (that is, normalized by the maximal average) can be understood as a first step in the direction of ergodic optimization as these can be used to identify the support of maximizing measures. A second breakthrough was obtained by Contreras [19] in the context of Lagrangian dynamics, which proves that $C^{2}$-generic hyperbolic Mañé sets contain a periodic orbit and that it actually reduces to a single periodic orbit in the case of surfaces.

The main goal here is to contribute to the ergodic optimization of singular-hyperbolic attractors in threedimensional manifolds, where the geometric Lorenz attractors form the paradigmatic examples. Although geometric Lorenz attractors admit a global cross-section, one cannot tackle this problem directly and to reduce their ergodic optimization to the ergodic optimization of their Poincare maps. Indeed, the presence of singularities makes not only the roof function to be piecewise smooth and unbounded, as the Poincaré return map is generally non-Markovian and just piecewise smooth with unbounded derivatives (cf. [2]). While one could expect the ideas in [29] to be useful to construct calibrated sub-actions in the previous context for a suitable (countable) Markov inducing scheme, a complete ergodic optimization description seems far from unattainable by this approach.

Our strategy to overcome the previous difficulties exploit the fact that singular-hyperbolic attractors can be approximated by horseshoes (see [2, 43]). Indeed, a singular-hyperbolic set with no singularities on a threedimensional manifold is uniformly hyperbolic (see e.g. [2]). On the one hand, the existence of Markov partitions for hyperbolic flows $[12,38]$ ensures that the suspended horseshoes can be modeled by suspension flows over a subshift of finite type. Then we prove that the ergodic optimization of hyperbolic flows can be reduced to the ergodic optimization of bilateral subshifts of finite type (and later to one-sided subshifts of finite type) with respect to induced observables. We prove that the previous reduction has a fibered structure in the space of observables, namely that is formed by submersions in the space of observables, and use the latter to prove that results on the ergodic optimization for bilateral subshifts of finite type lead to a translation of such results for suspended horseshoes (see Section 4). In particular, adapting [18, 34, 39] to the context of topologically mixing bilateral subshifts of finite type we prove that for each of these approximating suspended horseshoes: (i) there exists a open and dense set of Hölder observable with a unique maximizing measure, supported on a periodic orbit; (ii) there is a $G_{\delta}$-dense set of continuous observables with a unique maximizing measure, and it has zero entropy and full support; and (iii) there exists a $C^{0}$-dense subset formed by observables that admit uncountable many ergodic maximizing measures with positive entropy. Related results for the singular-hyperbolic attractors are obtained by an approximation argument, explored in Section 5.

Our main results can be grouped according to both the regularity and hyperbolicity of the flow, and the regularity of the observables.

Hyperbolic flows. Assume that $\left(X^{t}\right)_{t}$ is a $C^{1}$-flow and that $\Lambda$ is a hyperbolic basic set that is conjugated to a suspension flow over a subshift of finite type (see Subsection 2.2 for the definitions). We prove that typical continuous observables have unique and zero entropy maximizing measures. More precisely: 
Theorem A. Let $M$ be a d-dimensional compact boundaryless Riemannian manifold and $\left(X^{t}\right)_{t \in \mathbb{R}}$ be a $C^{1}$-flow in $M$. If $\Lambda \subset M$ is a hyperbolic basic set for $\left(X^{t}\right)_{t \in \mathbb{R}}$ that is conjugated to a suspension flow over a subshift of finite type then the following hold:

(1) there exists an open and dense set $O \subset C^{\alpha}(M, \mathbb{R})$ of observables $\varphi: M \rightarrow \mathbb{R}$ such that, for every $\varphi \in O$, there is a unique $\left(X^{t}\right)_{t}$-maximizing measure and it is supported on a periodic orbit;

(2) there exists a dense $G_{\delta}$ set $\mathcal{Z} \subset C^{0}(M, \mathbb{R})$ such that for every $\varphi \in \mathcal{Z}$, there is a single $\left(X^{t}\right)_{t \in \mathbb{R} \text {-maximizing }}$ measure, it has zero entropy and support equal to $\Lambda$; and

(3) there exists a dense set $\mathcal{D} \subset C^{0}(M, \mathbb{R})$ such that for every $\varphi \in \mathcal{D}$, there exists uncountably many

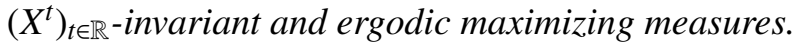

Since hyperbolic flows admit Markov partitions, these may be modeled by suspension flows. We observe that Theorem A will follow from a more general result on suspension flows (cf. Theorem 4.5).

Lorenz attractors. Our next results concern wild Lorenz attractors (we refer the reader to Subsection 2.3 for the definition). We prove the following.

Theorem B. Let $M$ be a 3-dimensional compact boundaryless Riemannian manifold and $\Lambda$ be a wild Lorenz attractor for a flow $\left(X^{t}\right)_{t}: M \rightarrow M$. Then:

(1) there exists a $C^{0}$-residual subset $\mathcal{R}_{1} \subset C^{0}(M, \mathbb{R})$ such that for every $\varphi \in \mathcal{R}_{1}$ there is an unique $\left(X^{t}\right)_{t^{-}}$ maximizing measure $\mu$ with respect to $\varphi$; moreover, $\mu$ is not atomic and the support supp $\mu$ contains the singularity;

(2) there is a $C^{\alpha}$-residual subset $\mathcal{R}_{2} \subset C^{\alpha}(M, \mathbb{R})$ of $\alpha$-Hölder observables such that, for every $\varphi \in \Re_{2}$ there is an unique $\left(X^{t}\right)_{t}$-maximizing measure $\mu$; moreover, either $\mu$ is supported on a critical element ( $a$ singularity or a periodic orbit) or it is non-atomic whose support contains some singularity.

One expects the previous result to hold for singular-hyperbolic attractors in general (see Subsection 2.2 for the definition). However, the argument in proof of Theorem B explores transitivity of locally maximal subsets and a characterization of the space of invariant measures for the wild Lorenz attractors (see e.g. Lemma 5.2). More precisely, the conclusion of Theorem B holds for all Lorenz attractors so that the set of periodic measures is dense in the convex space of invariant probabilities, a condition which holds for wild Lorenz attractors.

One other comment concerns invariant measures whose support contains the singularity at Theorem B. In general we cannot ensure that these measures are full supported on the attractor. Nevertheless, in the case of $C^{1}$-generic vector fields, we prove that every three-dimensional singular-hyperbolic attractor (including the Lorenz attractor) coincides with the closure of the unstable manifold of its singularities (cf. Proposition 2.11). Then, if we endow the space $\mathfrak{X}^{1}(M) \times C^{0}(M, \mathbb{R})$ with the product topology we have the following consequence:

Corollary 1. Let $M$ be a 3-dimensional compact boundaryless Riemannian manifold. There exists a Baire residual subset $\mathcal{R} \subset \mathfrak{X}^{1}(M) \times C^{0}(M, \mathbb{R})$ such that for any pair $(X, \varphi) \in \mathcal{R}$ there exists a unique $\left(X^{t}\right)_{t}$-maximizing measure $\mu$ with respect to $\varphi$ on each Lorenz attractor $\Lambda$ for the flow $\left(X^{t}\right)_{t}$ generated by $X$. Moreover, $\operatorname{supp} \mu=\Lambda$.

Some comments are in order. We could not rule out the possibility of having maximizing measures that are not supported at critical elements in item (2) of Theorem B. Furthermore, we observe that the condition that the support contains the singularity implies on a non-trivial recurrence to the singularity which can be thought as a replacement in this context to the fact that these measures are expected to have large support.

While this work was being written it was brought to our attention the results by Huang et al [25], which establish ergodic optimization for Axiom A flows using very different methods and establish a continuous-time Mañé-Conze-Guivarch-Bousch lemma. 
This paper is organized as follows. In Section 2 we give some preliminaries on ergodic optimization in the discrete time setting, suspension flows and weak forms of hyperbolicity for flows. In Section 3 we prove some results on the ergodic optimization for bilateral subshifts of finite type. The method explores a functional analytic description of the reduction using the solutions of the cohomological equation. A method for recovering results on the ergodic optimization for suspension semiflows from their counterpart for the Poincaré map is developed along Section 4, where we also prove Theorem A. In Section 5 we use that Lorenz attractors are approximated by horseshoes in order to characterize the space of invariant probabilities for Lorenz attractors and to prove Theorem B. Finally, some final comments are addressed in Section 6.

\section{Preliminaries}

2.1. Ergodic optimization for maps. In this subsection we recall some contributions for the ergodic optimization of maps. If $N$ is a compact metric space, $f: N \rightarrow N$ is a continuous map and $\psi: N \rightarrow \mathbb{R}$ is continuous, a maximizing measure for $f$ with respect to $\psi$ is an $f$-invariant Borel probability measure $\bar{\mu}$ which maximizes the integral of $\psi$ among all $f$-invariant Borel probabilities. In other words,

$$
\int \psi d \bar{\mu}=\max \left\{\int \psi d \bar{v}: \bar{v} \in \mathcal{M}_{1}(N, f)\right\} .
$$

We denote $M(\psi, f)=\max \left\{\int \psi d \bar{v}: \bar{v} \in \mathcal{M}_{1}(N, f)\right\}$. As discussed in the introduction there is a dichotomy depending on the regularity of the observables and structure of the underlying dynamics. The first results consider continuous maps with Bowen's specification property (see e.g. [8] for definition and a further discussion).

Theorem 2.1. [26, Theorem 3.2] Let $f: N \rightarrow N$ be a continuous map on a compact metric space $N$, and let $E$ be a topological vector space which is densely and continuously embedded in $C^{0}(N, \mathbb{R})$. Then the set of observables $\varphi \in E$ that have a unique maximizing measure is a countable intersection of open and dense subsets of $E$. In particular, if $E$ is a Baire space then the set above is dense in $E$.

Remark 2.2. Given $\alpha>0$, the previous theorem ensures that there exist Baire residual subsets in $C^{0}(N, \mathbb{R})$ and in $C^{\alpha}(N, \mathbb{R})$ formed by observables with a unique maximizing measure.

Remark 2.3. Theorem 2.1 admits a counterpart to continuous flows. Indeed, the argument in its proof relies on convergences both on the space of measures (in the weak* topology) and the space of (continuous) potentials, and it does not depend on the discrete-time or continuous-time nature of the dynamics itself. Thus, the set of observables having a unique maximizing measure (invariant by a continuous flow) forms a Baire generic subset on both the spaces of continuous and Hölder continuous observables.

By the previous discussion, for any fixed continuous flow $\left(X^{t}\right)_{t}$, typical observables have a unique maximizing measure. We would like to say more about these measures (e.g. to characterize the support of these measures). Before recalling such kind of results for maps we need a definition.

Definition 2.4. We say that $f$ satisfies the gluing orbit property if for any $\varepsilon>0$ there exists an integer $m=$ $m(\varepsilon) \geq 1$ so that for any $x_{1}, x_{2}, \ldots, x_{k} \in N$ and any integers $n_{1}, \ldots, n_{k} \geq 0$ there are $0 \leq p_{1}, \ldots, p_{k-1} \leq m(\varepsilon)$ and a point $y \in N$ so that $d\left(f^{j}(y), f^{j}\left(x_{1}\right)\right) \leq \varepsilon$ for every $0 \leq j \leq n_{1}$ and $d\left(f^{j+n_{1}+p_{1}+\cdots+n_{i-1}+p_{i-1}}(y), f^{j}\left(x_{i}\right)\right) \leq \varepsilon$ for every $2 \leq i \leq k$ and $0 \leq j \leq n_{i}$. If, in addition, $y \in N$ can be chosen periodic with period $\sum_{i=1}^{k}\left(n_{i}+p_{i}\right)$ for some $0 \leq p_{k} \leq m(\varepsilon)$ then we say that $f$ satisfies the periodic gluing orbit property.

The latter is a condition weaker than Bowen's specification and it is satisfied by transitive hyperbolic dynamics, and minimal equicontinuous maps, among other class of examples (cf. [7, 8] and references therein). 
Theorem 2.5. Let $f: N \rightarrow N$ be a continuous transformation of a compact metric space satisfying the periodic gluing orbit property. Then there is a dense $G_{\delta} \operatorname{set} Z \subset C^{0}(N, \mathbb{R})$ such that every $\varphi \in Z$ has a unique $\varphi$-maximizing measure, it has full support in $N$ and zero entropy.

Proof. This result is a simple modification of [34, Corollary 1.3]. Indeed, Corollary 1.2 in [34] ensures that the ergodic maximizing measures of a generic continuous function have the same properties as generic ergodic measures. Hence, it suffices to check that the gluing orbit property can replace Bowen's specification as a mechanism to prove that full supported and zero entropy measures are Baire generic.

Since the proof follows Sigmund's approach in [42] closely, with minor modifications, we just emphasize the diferences in the argument. Let $\left\{U_{i}\right\}_{i \geq 0}$ be a countable basis of the topology in $N$. For any $i \geq 0$, by weak* convergence it is clear that the space $\mathcal{E}_{i}$ of invariant probabilities $\mu$ so that $\mu\left(U_{i}\right)=0$ is closed. We claim that this set has also empty interior. Indeed, let $\mu \in \mathcal{M}_{1}(f)$ be such that $\mu\left(U_{i}\right)=0$, and let $\varepsilon>0$ and $\varphi_{i} \in C^{0}(N, \mathbb{R})$ be arbitrary and define the open neighborhood

$$
\mathcal{V}=\mathcal{V}\left(\mu ; \varphi_{1}, \ldots, \varphi_{k}, \frac{\varepsilon}{4}\right):=\left\{v \in \mathcal{M}_{1}(f):\left|\int \varphi_{i} d \mu-\int \varphi_{i} d v\right|<\frac{\varepsilon}{4}, \forall 1 \leq i \leq k\right\}
$$

of $\mu$, in the weak*-topology. By uniform continuity, there exists $\delta>0$ so that $\left|\varphi_{i}(x)-\varphi_{i}(y)\right|<\frac{\varepsilon}{4}$ whenever $d(x, y)<\delta$. Assume further, reducing $\delta$ is necessary, that there exists $x_{0} \in U_{i}$ so that $B\left(x_{0}, 2 \delta\right) \subset U_{i}$.

By Birkhoff's ergodic theorem there exists a $\mu$-full measure subset $N_{0} \subset N$ so that for every $x \in N_{0}$ and $1 \leq i \leq k$ the limit $\varphi_{i}^{*}(x)=\lim _{n \rightarrow \infty} \frac{1}{n} \sum_{j=0}^{n-1} \varphi_{i}\left(f^{j}(x)\right)$ exists and $\int \varphi_{i}^{*} d \mu=\int \varphi_{i} d \mu$. Let $\mathcal{P}$ be a finite partition of $N_{0}$ so that $\sup _{x \in P} \varphi_{i}^{*}(x)-\inf _{x \in P} \varphi_{i}^{*}(x)<\frac{\varepsilon}{4}$ for every $P \in \mathcal{P}$ and every $1 \leq i \leq k$. In consequence, for $x_{P} \in P$ arbitrary,

$$
\left|\int \varphi_{i} d \mu-\sum_{P \in \mathcal{P}} \mu(P) \varphi_{i}^{*}\left(x_{P}\right)\right|=\left|\int \varphi_{i}^{*} d \mu-\sum_{P \in \mathcal{P}} \mu(P) \varphi_{i}^{*}\left(x_{P}\right)\right|<\frac{\varepsilon}{4}
$$

Choose $N_{1} \geq 1$ so that $\left|\frac{1}{n} \sum_{j=0}^{n-1} \varphi_{i}\left(f^{j}\left(x_{P}\right)\right)-\int \varphi_{i} d \mu\right|<\frac{\varepsilon}{4}$ for every $1 \leq i \leq k$, every $P \in \mathcal{P}$ and every $n \geq N_{1}$. Choose $N_{2} \geq N_{1}$ so that every $m \geq N_{2}$ can be written as

$$
m=\sum_{P \in \mathcal{P}} m_{P} \quad \text { where } \quad\left|\frac{m_{P}}{m}-\mu(P)\right| \leq \frac{\varepsilon}{12[\# \mathcal{P}] \max _{1 \leq i \leq k}\left\|\varphi_{i}\right\|_{0}} .
$$

Finally, by the gluing orbit property, for each $N_{3} \geq N_{2}$ there exists a periodic point $z \in N$ so that $d\left(z, x_{0}\right)<\delta$ and for each $P \in \mathcal{P}$ the orbit of $z$ shadows the finite piece of orbit $\left\{x_{P}, f\left(x_{P}\right), \ldots, f^{N_{3}-1}\left(x_{P}\right)\right\}$ for $m_{P}$ consecutive times, recursively, with time lags in between the orbits of length at most $m(\delta)$ (here $m=m(\delta)$ is given by the gluing orbit property at scale $\delta$ ). The period of $z$ is

$$
\pi_{z}:=1+p_{0}+\sum_{j=1}^{\# \mathcal{P}}\left(N_{3}+p_{j}\right)
$$

where $0 \leq p_{j} \leq m(\delta)$ for every $0 \leq j \leq \# \mathcal{P}$. It is clear from the construction that $\mu_{z}:=\frac{1}{\pi_{z}} \sum_{j=0}^{\pi_{z}-1} \delta_{f^{j}(z)}$ is such that $\mu_{z}\left(U_{i}\right)>0$. Moreover, one can choose $N_{3} \gg N_{2}$ large enough so that the proportion of the orbit of the point $z$ outside of the shadowing process satisfies

$$
\frac{\sum_{j=0}^{\# \mathcal{P}} p_{j}}{1+p_{0}+\sum_{j=1}^{\# \mathcal{P}}\left(N_{3}+p_{j}\right)} \leq \frac{m(\delta) \# \mathcal{P}}{1+N_{3} \# \mathcal{P}}<\frac{\varepsilon}{12 \max _{1 \leq i \leq k}\left\|\varphi_{i}\right\|_{0}} .
$$

In particular, the proof that $\mu_{z} \in \mathcal{V}$ will follow the same lines of [42, pp. 104]. Altogether, the latter shows that each $\mathcal{E}_{i}$ is a closed set with empty interior, hence the invariant measures giving positive measure to open sets form a Baire generic subset. The proof that zero entropy measures form a Baire generic subset is identical and left as an exercise to the reader. 
We now focus on expanding dynamics. Given a compact metric space $N$, a map $T: N \rightarrow N$ is Ruelle expanding if there are $k \in \mathbb{Z}^{+}$and $0<\lambda<1$ such that for every point $x \in N$ there is a neighborhood $U_{x}$ of $x$ in $N$ and continuous branches $S_{i}, i=1, \ldots, \ell_{x} \leq k$ of the inverse of $T$ such that $T^{-1}\left(U_{x}\right)=\bigcup_{i=1}^{\ell_{x}} S_{i}\left(U_{x}\right), T \circ S_{i}=$ $I_{U_{x}}$ for all $i$, and $d\left(S_{i}(y), S_{i}(z)\right) \leq \lambda d(y, z)$ for all $y, z \in U_{x}$. Transitive Ruelle expanding maps satisfy the periodic gluing orbit property [7]. Throughout, assume without loss of generality that diam $N=1$ and let $C^{\alpha}(N, \mathbb{R})$ denote the space of $\alpha$-Hölder observables (ie. $\varphi \in C^{\alpha}(N, \mathbb{R})$ if there are constants $C, \alpha>0$ so that $|\varphi(x)-\varphi(y)| \leq C d(x, y)^{\alpha}$ for all $\left.x, y \in N\right)$.

Theorem 2.6. [18] If $N$ is a compact metric space and $f: N \rightarrow N$ is a Ruelle expanding map there is an open and dense set $O \subset C^{\alpha}(N, \mathbb{R})$ so that every $\varphi \in O$ admits a unique $\varphi$-maximizing measure and it is supported on a periodic orbit.

Remark 2.7. As stated above, Theorem 2.6 differs from the version presented in [18], which was stated for the space $\operatorname{Lip}(N, \mathbb{R})$ of Lipschitz observables instead of $C^{\alpha}(N, \mathbb{R})$. Nevertheless, Theorem 2.6 is a direct consequence of the main result in [18] together with the fact that, since diam $N=1$, the space of $\alpha$-Hölder continuous functions with respect to the metric $d(\cdot, \cdot)$ coincides with the space of Lipschitz functions with respect to the modified metric $d_{\alpha}(\cdot, \cdot)=d(\cdot, \cdot)^{\alpha}$.

Finally we recall Shinoda's result on the dense non-uniqueness of maximizing measures.

Theorem 2.8. [39] Let $\left(\Sigma_{R}, \sigma\right)$ be a one-sided topologically mixing subshift of finite type. There exists a dense subset $\mathcal{D} \subset C^{0}\left(\Sigma_{R}, \mathbb{R}\right)$ such that for every $\varphi \in \mathcal{D}$ there exist uncountably many ergodic $\varphi$-maximizing measures with full support and positive entropy.

2.2. Hyperbolic flows. Let $M$ be a closed Riemannian manifold and let $\left(X^{t}\right)_{t}: M \rightarrow M$ be a smooth flow. Let $\Lambda \subseteq M$ be a compact $\left(X^{t}\right)_{t}$-invariant set. The flow $\left(X^{t}\right)_{t}: \Lambda \rightarrow \Lambda$ is uniformly hyperbolic if for every $x \in \Lambda$ there exists a $D X^{t}$-invariant and continuous splitting $T_{x} M=E_{x}^{s} \oplus E_{x}^{X} \oplus E_{x}^{u}$ and constants $C>0$ and $0<\theta_{1}<1$ such that

$$
\left\|D X^{t} \mid E_{x}^{s}\right\| \leq C \theta_{1}^{t} \quad \text { and } \quad\left\|\left(D X^{t}\right)^{-1} \mid E_{x}^{u}\right\| \leq C \theta_{1}^{t},
$$

for every $t \geq 0$. We say that $\left(X^{t}\right)_{t}$ is an Anosov flow if $\left(X^{t}\right)_{t}: M \rightarrow M$ is uniformly hyperbolic. It is well known that adapted metrics exist, hence we may assume $C=1$. Given a hyperbolic set $\Lambda$, for each $x \in \Lambda$ consider the stable and the unstable manifolds $W^{s}(x)=\left\{y \in M: \operatorname{dist}\left(X^{t}(y), X^{t}(x)\right) \rightarrow 0\right.$ as $\left.t \rightarrow+\infty\right\}$ and $W^{u}(x)=\left\{y \in M: \operatorname{dist}\left(X^{t}(y), X^{t}(x)\right) \rightarrow 0\right.$ as $\left.t \rightarrow-\infty\right\}$, respectively. By the stable manifold theorem, uniform hyperbolicity ensures that there exists $\varepsilon>0$ so that the largest connected components $W_{l o c}^{s}(x) \subset W^{s}(x)$ and $W_{l o c}^{u}(x) \subset W^{u}(x)$ of size $\varepsilon$ containing $x$ are smooth submanifolds, called respectively (local) stable and unstable manifolds (of size $\varepsilon$ ) at the point $x$. Moreover:

(1) $T_{x} W_{l o c}^{s}(x)=E^{s}(x)$ and $T_{x} W_{l o c}^{u}(x)=E^{u}(x)$;

(2) for each $t>0$ we have $X^{t}\left(W_{l o c}^{s}(x)\right) \subset W_{l o c}^{s}(x)\left(X^{t}(x)\right)$ and $X^{-t}\left(W_{l o c}^{u}(x)\right) \subset W_{l o c}^{u}\left(X^{-t}(x)\right)$;

(3) there exist $\kappa>0$ and $\gamma \in(0,1)$ such that for each $t>0$ we have $d\left(X^{t}(y), X^{t}(x)\right) \leq \kappa \gamma^{t} d(y, x)$ for $y \in$ $W_{l o c}^{s}(x)$, and $d\left(X^{-t}(y), X^{-t}(x)\right) \leq \kappa \gamma^{t} d(y, x)$ for $y \in W_{l o c}^{u}(x)$.

Moreover, the set $\Lambda$ is locally maximal if there exists an open neighborhood $U$ of $\Lambda$ such that $\Lambda=\bigcap_{t \in \mathbb{R}} X^{t}(U)$.

A point $p \in M$ is a singularity for $X$ if $X(p)=0$, and is called a regular point otherwise. We say that a singularity $p$ is hyperbolic if the one-point invariant set $\{p\}$ is a hyperbolic set. A point $p \in M$ is periodic if there exists a minimum period $T>0$ so that $X^{T}(p)=p$, and we say that $p$ is a periodic hyperbolic point if the orbit $O(p)=\cup_{t \in[0, T]} X^{t}(p)$ is a hyperbolic set for $X$. Finally, (an orbit of) a point $x$ by the flow is called a critical element if it is either periodic or $x$ is a singularity. 
Now let $\Lambda$ be a locally maximal hyperbolic set. For any sufficiently small $\varepsilon$, there exists a $\delta>0$ such that if $x, y \in \Lambda$ are at a distance $d(x, y) \leq \delta$, then there exists a unique $t=t(x, y) \in[-\varepsilon, \varepsilon]$ for which the set $[x, y]=W_{l o c}^{s}(x)\left(X^{t}(x)\right) \cap W_{l o c}^{u}(y)$ is a single point in $\Lambda$ (see e.g. [23, Proposition 7.2]).

Definition 2.9. We say that $\Lambda$ is a hyperbolic basic set if (i) $\Lambda$ is hyperbolic (not a fixed point); (ii) the periodic orbits of $\left(X^{t}\right)_{t} \mid \Lambda$ are dense in $\Lambda$; (iii) $\left(X^{t}\right)_{t} \mid \Lambda$ is transitive (contains a dense orbit); (iv) $\Lambda$ is locally maximal. We say $\left(X^{t}\right)_{t}$ is Axiom $A$ if $\Omega$ is the disjoint union of hyperbolic sets and a finite number of hyperbolic fixed points. Moreover, we say that $\Lambda$ is a horseshoe if it is topologically conjugated to the suspension flow over a subshift of finite type.

2.3. Singular-hyperbolic and Lorenz attractors. We say that a compact $\left(X^{t}\right)_{t \in \mathbb{R}}$-invariant set $\Lambda \subset M$ is partially hyperbolic if there are a continuous invariant splitting $T_{\Lambda} M=E^{s} \oplus E^{c}$, constants $C>0$ and $\lambda \in(0,1)$ so that

$$
\left\|D_{x} X^{t} \mid E_{x}^{s}\right\| \leq C \lambda^{t} \quad \text { and } \quad\left\|D_{x} X^{t}\left|E_{x}^{s}\|\cdot\| D_{X^{t}(x)} X_{-t}\right| E_{X^{t}(x)}^{c}\right\| \leq C \lambda^{t}
$$

for every $x \in \Lambda$ and $t \geq 0$. If, in addition, the following two conditions (i) and (ii) hold, then we say that $\Lambda$ is sectional-hyperbolic:

(i) every singularity $p \in \Lambda$ is hyperbolic;

(ii) $E^{c}$ is sectionally expanding, i.e. $\operatorname{dim} E^{c} \geq 2$ and $\left|\operatorname{det}\left(D_{x} X^{t} \mid L_{x}\right)\right| \geq C^{-1} \lambda^{t}$ for every $x \in \Lambda, t \geq 0$, and every two-dimensional subspace $L_{x} \subset E_{x}^{c}$.

With some abuse of notation, we say that the flow $\left(X^{t}\right)_{t \in \mathbb{R}}$ is partially hyperbolic if $M$ is a partially hyperbolic set. $\Lambda$ is said to be singular-hyperbolic if $\Lambda$ is partially hyperbolic such that it satisfies the above condition (i) and $E^{c}$ is volume expanding, i.e. $\left|\operatorname{det}\left(D_{x} X^{t} \mid E_{x}^{c}\right)\right| \geq C^{-1} \lambda^{t}$ for every $x \in \Lambda, t \geq 0$.

Finally we give a brief description of the construction of geometric Lorenz attractors. We will follow [2] (see also [3, Section 3]). Let $\Sigma=\left\{(x, y, 1) \in \mathbb{R}^{3}:|x|,|y| \leq 1\right\}$ and $\Gamma=\left\{(0, y, 1) \in \mathbb{R}^{3}:|y| \leq 1\right\}$. Consider a $C^{1}$-vector field $X_{0}$ on $\mathbb{R}^{3}$ so that the following conditions hold:

(1) For any point $(x, y, z)$ in a neighborhood of the origin $(0,0,0)$ of $\mathbb{R}^{3}, X_{0}$ is given by

$$
(\dot{x}, \dot{y}, \dot{z})=\left(\lambda_{1} x,-\lambda_{2} y,-\lambda_{3} z\right)
$$

where $0<\lambda_{3}<\lambda_{1}<\lambda_{2}$.

(2) All forward orbits of $X$ starting from $\Sigma \backslash \Gamma$ will return to $\Sigma$ and the first return map $P: \Sigma \backslash \Gamma \rightarrow \Sigma$ is a piecewise $C^{1}$-diffeomorphism which has the form

$$
P(x, y, 1)=(\alpha(x), \beta(x, y), 1),
$$

where $\alpha:[-1,1] \backslash\{0\} \rightarrow[-1,1]$ is a piecewise $C^{1}$-map with $\alpha(-x)=-\alpha(x)$ and there is $0<\gamma<1$ satisfying

$$
\begin{cases}\alpha(x)=x^{\gamma} & \text { in a neighborhood of } 0 \\ \alpha^{\prime}(x)>\sqrt{2}, & \text { for any } x \neq 0 \\ \alpha(1)<1, & \\ \lim _{x \rightarrow 0^{+}} \alpha(x)=-1, & \\ \lim _{x \rightarrow 0^{+}} \alpha(x)=\infty, & \end{cases}
$$

and there exists $\lambda \in(0,1)$ so that $\left|\frac{\partial \beta}{\partial y}(x, y)\right| \leq \lambda$ for every $(x, y, 1) \in \Sigma$.

The second condition in (2.3) ensures that the one-dimensional map $\alpha:[-1,1] \backslash\{0\} \rightarrow[-1,1]$ is locally eventually onto: for any open interval $I \subset[-1,1] \backslash\{0\}$ there exists $N \geq 1$ so that $\alpha^{N}(I)=(\alpha(-1), \alpha(1)$ ). (cf. [2, Lemma 3.16]). We say that a one-dimensional Lorenz map is wild if $\sup _{\mu} \int \log \left|f^{\prime}\right| d \mu=+\infty$, where the supremum is taken over all $f$-invariant probability measures $\mu$. 
Remark 2.10. Although the derivative of Lorenz maps is unbounded it can occur that for a particular Lorenz map $\alpha$ all orbits have slow recurrence to the singular point, causing all invariant measures to have uniformly bounded Lyapunov exponent (e.g. this is the case when the singular point is pre-periodic repelling). While it is expected for both classes of wild and non-wild geometric Lorenz attractors to be locally dense (in a $C^{2}$ neighborhood of the original vector field), it was recently announced that non-wild Lorenz maps are actually generic along special parameterized families of Lorenz attractors [35].

There exists an open elipsoid $V \subset \mathbb{R}^{3}$ containing the origin such that the vector field $X_{0}$ points inwards, hence it exhibits an attractor. If $\mathcal{U} \subset \mathfrak{X}^{1}\left(\mathbb{R}^{3}\right)$ is a $C^{1}$-open set of the vector field $X_{0}$ and an open elipsoid $V \subset \mathbb{R}^{3}$ containing the origin such that every $X \in \mathcal{U}$ exhibits a partially hyperbolic attractor $\Lambda_{X}=\bigcap_{t \geq 0} \overline{X^{t}(V)}$, and it is called geometric Lorenz attractor. We say that $\Lambda$ is a wild Lorenz attractor if the corresponding one-dimensional Lorenz map $\alpha$, obtained by quotient along local stable leaves in the cross-section $\Sigma$, is wild.

It is well known that for every $X \in \mathcal{U}$ there exists a periodic point $p_{X} \in \Lambda_{X}$ so that the Lorenz attractor $\Lambda_{X}$ coincides with the homoclinic class $H\left(p_{X}\right):=\overline{W^{s}\left(p_{X}\right) \pitchfork W^{u}\left(p_{X}\right)}$ (cf. [2, Proposition 3.17]). In particular, the attractor is transitive and, by Birkhoff-Smale's theorem (see e.g. [28]) it admits a dense set of hyperbolic periodic orbits. Moreover, any singular-hyperbolic attractor without singularities is uniformly hyperbolic (see e.g. [2]).

As three-dimensional singular-hyperbolic attractors have only hyperbolic singularities whose unstable manifolds are one-dimensional (see e.g. [2]) we denote by $W^{u,+}(\sigma)$ and $W^{u,-}(\sigma)$ the connected components of $W^{u}(\sigma) \backslash\{\sigma\}$. We finish this subsection with the following characterization for $C^{1}$-generic singular-hyperbolic attractors.

Proposition 2.11. There is a residual subset $\mathcal{R} \subset \mathfrak{X}^{1}(M)$ such that if $\Lambda$ is a singular-hyperbolic attractor for $X \in \mathcal{R}$ and $\Lambda \cap \operatorname{Sing}(X) \neq \emptyset$ then $\Lambda=\overline{W^{u,+}(\sigma)}=\overline{W^{u,-}(\sigma)}$ for every $\sigma \in \Lambda \cap \operatorname{Sing}(X)$.

Proof. This results is a consequence of Theorem 4.2 in [32]. Indeed, the argument in [32, pp 372-373] uses the $C^{1}$-connecting lemma in order to prove that $\overline{W^{u}(\sigma)}$ is a Lyapunov stable set for every singularity $\sigma \in \Lambda \cap \operatorname{Sing}(X)$ of a $C^{1}$-generic vector field $X$. Nevertheless, the argument follows without changes for the set $\overline{W^{u,+}(\sigma)}$ (and $\left.\overline{W^{u,-}(\sigma)}\right)$ instead of $\overline{W^{u}(\sigma)}$. Hence, there exists a $C^{1}$-residual subset $\mathcal{R}_{*} \subset \mathfrak{X}^{1}(M)$ so that if $\Lambda$ is a singularhyperbolic attractor for $X \in \mathcal{R}_{*}$ and $\Lambda \cap \operatorname{Sing}(X) \neq \emptyset$ then $\Lambda=\overline{W^{u, *}(\sigma)}$ for every $\sigma \in \Lambda \cap \operatorname{Sing}(X)$, for $* \in\{+,-\}$. The $C^{1}$-residual subset $\mathcal{R}=\mathcal{R}_{+} \cap \mathcal{R}_{-}$satisfies the requirements of the proposition.

Remark 2.12. All non-atomic ergodic measures for singular-hyperbolic attractors can be approximated by periodic measures due to the shadowing lemma in [40]. Indeed, by the presence of singularities the only difference is that the closing lemma should be replaced by the extended Liao closing lemma in [22]. We are grateful to X. Tian for pointing out this fact to us.

2.4. Suspension semiflows. Let $f: N \rightarrow N$ be a continuous map on a compact metric space $\left(N, d_{N}\right)$ and let $r: N \rightarrow(0, \infty)$ be a continuous function bounded away from zero. Consider the quotient space

$$
N^{r}=\{(x, t): 0 \leq t \leq r(x), x \in N\} / \sim
$$

where $(x, r(x)) \sim(f(x), 0)$. The suspension semiflow over $f$ with height function $r$ is the semiflow $\left(X^{t}\right)_{t \in \mathbb{R}_{+}}$in $N^{r}$ with $X^{t}: N^{r} \rightarrow N^{r}$ defined by $X^{t}(x, s)=\left(f^{n}(x), s^{\prime}\right)$, where $n$ and $s^{\prime}$ are uniquely determined by $\sum_{i=0}^{n-1} r\left(f^{i}(x)\right)+$ $s^{\prime}=t+s$ and $0 \leq s^{\prime}<r\left(f^{n}(x)\right)$. If $f$ is a homeomorphism then the previous expression defines a flow.

We recall Bowen and Walters distance for suspension flows [14]. Assume without loss of generality that the diameter of $N$ is bounded by one. We first assume that the height function $r$ is constant equal to 1 . Given $x, y \in N$ and $t \in[0,1]$, we define the length of the horizontal segment $[(x, t),(y, t)]$ by

$$
\rho_{h}((x, t),(y, t))=(1-t) d_{N}(x, y)+t d_{N}(f(x), f(y)) .
$$


Clearly, $\rho_{h}((x, 0),(y, 0))=d_{N}(x, y)$ e $\rho_{h}((x, 1),(y, 1))=d_{N}(f(x), f(y))$. Moreover, given points $(x, t),(y, s) \in N^{r}$ in the same orbit, we define the length of the vertical segment $[(x, t),(y, s)]$ by

$$
\rho_{v}((x, t),(y, s))=\inf \left\{|q|: X^{q}(x, t)=(y, s) \text { e } q \in \mathbb{R}\right\} .
$$

For the height function $r=1$, the Bowen-Walters distance $d((x, t),(y, s))$ between two points $(x, t),(y, s) \in N^{r}$ is defined as the infimum of the lengths of all paths between $(x, t)$ and $(y, s)$ that are composed of finitely many horizontal and vertical segments. Now we consider an arbitrary continuous height function $r: N \rightarrow(0, \infty)$ and we introduce the Bowen-Walters distance $d_{N^{r}}$ in $N^{r}$.

Definition 2.13. Given $(x, t),(y, s) \in N^{r}$, we define

$$
d_{N^{r}}((x, t),(y, s))=d((x, t / r(x)),(y, s / r(y))),
$$

where $d$ is the Bowen-Walters distance for the height function $r=1$.

For an arbitrary function $r$, a horizontal segment takes the form $w=[(x, t \cdot r(x)),(y, t \cdot r(y))]$, and its length is given by $\ell_{h}(w)=(1-t) d_{N}(x, y)+t d_{N}(f(x), f(y))$. Moreover, the length of a vertical segment $w=[(x, t),(x, s)]$ is now $\ell_{v}(w)=|t-s| / r(x)$, for any sufficiently close $t$ and $s$. It is sometimes convenient to measure distances in another manner. Namely, given $(x, t),(y, s) \in N^{r}$, let

$$
d_{\pi}((x, t),(y, s))=\min \left\{\begin{array}{c}
d_{N}(x, y)+|t-s| \\
d_{N^{r}}(f(x), y)+r(x)-t+s \\
d_{N^{r}}(x, f(y))+r(y)-s+t
\end{array}\right\} .
$$

Although $d_{\pi}$ may not be a distance it is related to the Bowen-Walters distance.

Proposition 2.14. [6, Proposition 2.1] If $f$ is an invertible Lipschitz map with Lipschitz inverse, then there exists a constant $c \geq 1$ such that $c^{-1} d_{\pi}(p, q) \leq d_{N^{r}}(p, q) \leq c d_{\pi}(p, q)$ for every $p, q \in N^{r}$.

Remark 2.15. Given a $\left(X^{t}\right)_{t}$-invariant measure $\mu$ there exists an $f$-invariant probability $\tilde{\mu}$ on $N$ such that $\mu=\tilde{\mu} \times$ $L e b / \int r d \tilde{\mu}$. It is well known that if $r$ is bounded away from zero then $\tilde{\mu} \mapsto \tilde{\mu} \times L e b / \int r d \tilde{\mu}$ is a bijection between the space $\mathcal{M}_{1}(N, f)$ of $f$-invariant probabilities and the space $\mathcal{M}_{1}\left(N^{r},\left(X^{t}\right)_{t}\right)$ of $\left(X^{t}\right)_{t}$-invariant probabilities.

\section{ERGODIC OPTIMIZATION FOR BILATERAL SUBSHIFTS}

3.1. Symbolic dynamics. Let $\Sigma_{n}=\{1, \ldots, n\}^{\mathbb{Z}}$ be the space of all sequences $\underline{x}=\left\{x_{i}\right\}_{i=-\infty}^{\infty}$ with $x_{i} \in\{1, \ldots, n\}$ for all $i \in \mathbb{Z}$. We define the (left) shift homeomorphism $\sigma: \Sigma_{n} \rightarrow \Sigma_{n}$ by $\sigma\left(\left\{x_{i}\right\}_{i=-\infty}^{\infty}\right)=\left\{x_{i+1}\right\}_{i=-\infty}^{\infty}$. If $\mathbf{R}$ is a $n \times n$ transition matrix formed by 0 's and 1 's, and

$$
\Sigma_{\mathbf{R}}=\left\{\underline{x} \in \Sigma_{n}: \mathbf{R}_{x_{i} x_{i+1}}=1 \text { for all } i \in \mathbb{Z}\right\},
$$

we say $\sigma: \Sigma_{\mathbf{R}} \rightarrow \Sigma_{\mathbf{R}}$ a subshift of finite type (determined by $\mathbf{R}$ ). We denote by $\Sigma_{n}^{+}=\{1, \ldots, n\}^{\mathbb{N}}$ the space of all sequences $\underline{x}=\left\{x_{i}\right\}_{i=0}^{\infty}$ with $x_{i} \in\{1, \ldots, n\}$ for all $i \in \mathbb{N}$ and define the one-sided (left) shift homeomorphism $\sigma: \Sigma_{n}^{+} \rightarrow \Sigma_{n}^{+}$by $\sigma\left(\left\{x_{i}\right\}_{i=0}^{\infty}\right)=\left\{x_{i+1}\right\}_{i=0}^{\infty}$. One-sided subshifts of finite type are defined analogously. It is easy to check that a one-sided subshift of finite type is a Ruelle expanding map.

For $\varphi: \Sigma_{\mathbf{R}} \rightarrow \mathbb{R}$ continuous we define the variation of $\varphi$ on $k$-cylinders by

$$
\operatorname{var}_{k} \varphi=\sup \left\{|\varphi(\underline{x})-\varphi(\underline{y})|: x_{i}=y_{i} \text { for all }|i| \leq k\right\} .
$$

Let $\mathscr{F}_{\mathbf{R}}$ be the family of all continuous observables $\varphi: \Sigma_{\mathbf{R}} \rightarrow \mathbb{R}$ for which there exists positive constants $b$ and $c \in(0,1)$ so that $\operatorname{var}_{k} \varphi \leq b c^{k}$ for all $k \geq 0$. 
Remark 3.1. For any $\beta \in(0,1)$ one can define the metric $d_{\beta}$ on $\Sigma_{\mathbf{R}}$ by $d_{\beta}(\underline{x}, y)=\beta^{N}$ where $N$ is the largest non-negative integer with $x_{i}=y_{i}$ for every $|i|<N$. Then $\mathscr{F}_{\mathbf{R}}$ is the set of functions which have a positive Hölder exponent with respect to $d_{\beta}$. In fact, for $\underline{x}, y \in \Sigma_{\mathbf{R}}$ there is $N \in \mathbb{N}$ such that $d_{\beta}(x, y)=\beta^{N}$, this means that $\underline{x}$ and $\underline{y}$ are in the same $N$-cylinder and any $\varphi \in \overline{\mathscr{F}}_{\mathbf{R}}$ satisfies $\operatorname{var}_{N} \varphi \leq b c^{N}$, which implies $|\varphi(\underline{x})-\varphi(y)| \leq b c^{N}$. Choosing $\alpha \in(0,1)$ such that $c \leq \beta^{\alpha}$ we have $|\varphi(\underline{x})-\varphi(\underline{y})| \leq b\left(\beta^{N}\right)^{\alpha}=b d_{\beta}(\underline{x}, \underline{y})^{\alpha}$. This means that $\varphi$ is $\alpha$-Hölder in the metric $d_{\beta}$.

3.2. From unilateral to bilateral subshifts of finite type. Here we build over the results for expanding maps in Subsection 2.1 and the following classical result (see e.g. [13, Lemma 1.6]) on solutions of the cohomological equation, to consider the ergodic optimization of bilateral subshifts of finite type.

Lemma 3.2. If $\varphi \in \mathscr{F}_{\mathbf{R}}$, then there exists a continuous function $u=u_{\varphi}: \Sigma_{\mathbf{R}} \rightarrow \mathbb{R}$ such that $\psi:=\varphi+u \circ \sigma-u \in$ $\mathscr{F}_{\mathbf{R}}$ and $\psi(\underline{x})=\psi(\underline{y})$ whenever $x_{i}=y_{i}$ for all $i \geq 0$.

Proof. Although this is well known lemma, we include its proof for the reader's convenience, as we shall need the expression for the solution of the cohomological equation. For each $1 \leq t \leq n$ pick $\left\{a_{k, t}\right\}_{k=-\infty}^{\infty} \in \Sigma_{R}$ with $a_{0, t}=t$. Define $\rho: \Sigma_{R} \rightarrow \Sigma_{R}$ by $\rho(\underline{x})=\underline{x}^{*}$, where $x_{k}^{*}=x_{k}$ for $k>0$ and $x_{k}^{*}=a_{k, x_{0}}$ for $k \leq 0$. Let

$$
u(\underline{x})=\sum_{j=0}^{\infty}\left(\varphi\left(\sigma^{j}(\underline{x})\right)-\varphi\left(\sigma^{j}(\rho(\underline{x}))\right)\right) .
$$

Note that $\left.\mid \varphi\left(\sigma^{j}(\underline{x})\right)-\varphi\left(\sigma^{j} \rho(\underline{x})\right)\right) \mid \leq \operatorname{var}_{j} \varphi \leq b \alpha^{j}$ because $\sigma^{j}(\underline{x})$ and $\sigma^{j}(\rho(\underline{x}))$ agree in places from $-j$ to $+\infty$. As $\sum_{j=0}^{\infty} b \alpha^{j}<\infty$, the function $u$ is well defined and continuous. If $x_{i}=y_{i}$ for all $|i| \leq n$, then, for $j \in[0, n]$, $\left|\varphi\left(\sigma^{j}(\underline{x})\right)-\varphi\left(\sigma^{j}(\underline{y})\right)\right| \leq \operatorname{var}_{n-j} \varphi \leq b \alpha^{n-j}$ and $\left|\varphi\left(\sigma^{j} \rho((\underline{x}))\right)-\varphi\left(\sigma^{j} \rho((\underline{y}))\right)\right| \leq b \alpha^{n-j}$. Hence

$$
\begin{aligned}
|u(\underline{x})-u(\underline{y})| & \leq \sum_{j=0}^{\left[\frac{n}{2}\right]}\left|\varphi\left(\sigma^{j}(\underline{x})\right)-\varphi\left(\sigma^{j}(\underline{y})\right)+\varphi\left(\sigma^{j} \rho((\underline{x}))\right)-\varphi\left(\sigma^{j} \rho((\underline{y}))\right)\right|+2 \sum_{j>\left[\frac{n}{2}\right]} \alpha^{j} \\
& \leq 2 b\left(\sum_{j=0}^{\left[\frac{n}{2}\right]} \alpha^{n-j}+\sum_{j>\left[\frac{n}{2}\right]} \alpha^{j}\right) \leq \frac{4 b \alpha\left[\frac{n}{2}\right]}{1-\alpha} .
\end{aligned}
$$

This shows that $u \in \mathscr{F}_{\mathbf{R}}$. Hence the function $\psi:=\varphi+u \circ \sigma-u$ belongs to $\mathscr{F}_{\mathbf{R}}$ and it satisfies

$$
\begin{aligned}
\psi(\underline{x}) & =\varphi(\underline{x})+\sum_{j=-1}^{\infty}\left(\varphi\left(\sigma^{j+1} \rho((\underline{x}))\right)-\varphi\left(\sigma^{j+1}(\underline{x})\right)\right)+\sum_{j=0}^{\infty}\left(\varphi\left(\sigma^{j+1}(\underline{x})\right)-\varphi\left(\sigma^{j}(\rho(\underline{x}))\right)\right) \\
& =\varphi(\sigma \rho((\underline{x})))+\sum_{j=0}^{\infty}\left(\varphi\left(\sigma^{j+1}(\underline{x})\right)-\varphi\left(\sigma^{j}(\rho(\underline{x}))\right)\right) .
\end{aligned}
$$

The final expression in the right-hand side above depends only on $\left\{x_{i}\right\}_{i=0}^{\infty}$, as desired.

Now we analyze the coboundary map (3.1) as a function of the observable. By Remark 3.1, up to a change of metric we have that the space $\mathscr{F}_{\mathbf{R}}$ coincides with the space of Hölder continuous observables. Hence we have the following:

Lemma 3.3. Let $D^{+}$be the set of observables $\psi \in C^{\alpha}\left(\Sigma_{\mathbf{R}}, \mathbb{R}\right)$ so that $\psi(\underline{x})=\psi(\underline{y})$ whenever $x_{i}=y_{i}$ for all $i \geq 0$. Then the map $\Xi: C^{\alpha}\left(\Sigma_{\mathbf{R}}, \mathbb{R}\right) \rightarrow D^{+}$given by $\Xi(\varphi)=\varphi+u \circ \sigma-u$, where $u=\bar{u}_{\varphi}: \Sigma_{\mathbf{R}} \rightarrow \mathbb{R}$ is given by Lemma 3.2 , is a submersion. 
Proof. A simple computation shows that the map $u: C^{\alpha}\left(\Sigma_{\mathbf{R}}, \mathbb{R}\right) \rightarrow C^{\alpha}\left(\Sigma_{\mathbf{R}}, \mathbb{R}\right)$ given by $u(\varphi)(\underline{x})=\sum_{j=0}^{\infty}\left(\varphi\left(\sigma^{j}(\underline{x})\right)-\right.$ $\left.\varphi\left(\sigma^{j}(\rho(\underline{x}))\right)\right)$ is well defined and linear, hence $\Xi: C^{\alpha}\left(\Sigma_{\mathbf{R}}, \mathbb{R}\right) \rightarrow D^{+}$is also linear. As $\Xi$ is surjective, by construction, we conclude that $\Xi: C^{\alpha}\left(\Sigma_{\mathbf{R}}, \mathbb{R}\right) \rightarrow D^{+}$is a submersion.

Remark 3.4. If $\varphi \in C^{\alpha}\left(\Sigma_{\mathbf{R}}, \mathbb{R}\right)$ the observable $\tilde{\varphi} \in C^{\alpha}\left(\Sigma_{\mathbf{R}}, \mathbb{R}\right)$ defined by $\tilde{\varphi}\left(\left\{x_{i}\right\}_{i=-\infty}^{\infty}\right)=\varphi\left(\left\{x_{i}\right\}_{i=0}^{\infty}\right)$ is constant along local stable leaves. Reciprocally, if $\tilde{\varphi} \in C^{\alpha}\left(\Sigma_{\mathbf{R}}, \mathbb{R}\right)$ satisfies $\tilde{\varphi}(\underline{x})=\tilde{\varphi}(\underline{y})$ whenever $x_{i}=y_{i}$ for all $i \geq 0$, then one can associate an observable in $C^{\alpha}\left(\Sigma_{\mathbf{R}}^{+}, \mathbb{R}\right)$ by $\varphi\left(\left\{x_{i}\right\}_{i=0}^{\infty}\right)=\tilde{\varphi}\left(\left\{x_{i}\right\}_{i=-\infty}^{\infty}\right)$. The functions in $C^{\alpha}\left(\Sigma_{\mathbf{R}}^{+}, \mathbb{R}\right)$ are thus identified with the subclass of $C^{\alpha}\left(\Sigma_{\mathbf{R}}, \mathbb{R}\right)$ formed by functions that are constant on local stable leaves. Indeed, given the identification $\Sigma_{\mathbf{R}}^{+} \simeq \Sigma_{\mathbf{R}} / \sim$, where $\underline{x} \sim \underline{y}$ if $x_{i}=y_{i}$ for all $i \geq 0$ and $\underline{x}, \underline{y} \in \Sigma_{\mathbf{R}}$, one can identify $C^{\alpha}\left(\Sigma_{\mathbf{R}}^{+}, \mathbb{R}\right) \simeq C^{\alpha}\left(\Sigma_{\mathbf{R}}, \mathbb{R}\right) / \sim \simeq D^{+}$.

The next proposition is the main result in this subsection, and it extends Theorem 2.6 for bilateral subshifts of finite type.

Proposition 3.5. There is an open and dense subset of $\mathcal{R} \subset C^{\alpha}\left(\Sigma_{\mathbf{R}}, \mathbb{R}\right)$ such that every $\varphi \in \mathcal{R}$ admits a unique $\varphi$-maximizing measure and it is supported on a periodic orbit of $\sigma: \Sigma_{\mathbf{R}} \rightarrow \Sigma_{\mathbf{R}}$.

Proof. Since a transitive one-sided subshift of finite type is a Ruelle expanding map we can apply Theorem 2.6 to $\sigma: \Sigma_{\mathbf{R}}^{+} \rightarrow \Sigma_{\mathbf{R}}^{+}$and obtain an open and dense set $O \subset C^{\alpha}\left(\Sigma_{\mathbf{R}}^{+}, \mathbb{R}\right)$ such that for each $\varphi \in O$ there is a single $\varphi$-maximizing measure and it is supported on a periodic orbit. By the isomorphism in Remark 3.4, there exists an open and dense set $O^{+} \subset D^{+}$such that every $\varphi \in O^{+}$has a single $\varphi$-maximizing measure and it is supported on a periodic orbit. In fact, for every $\sigma$-invariant probability $\mu$ in $\Sigma_{\mathbf{R}}^{+}$there is a natural way to associate a unique invariant probability $\tilde{\mu}$ on $\Sigma_{R}$. Following [13, Section C], for $\varphi \in C^{0}\left(\Sigma_{\mathbf{R}}^{+}, \mathbb{R}\right)$ define $\varphi^{*} \in C^{0}\left(\Sigma_{\mathbf{R}}^{+}, \mathbb{R}\right)$ by

$$
\varphi^{*}\left(\left\{x_{i}\right\}_{i=0}^{\infty}\right):=\min \left\{\varphi(\underline{y}): \underline{y} \in \Sigma_{\mathbf{R}}, y_{i}=x_{i} \text { for all } i \geq 0\right\} .
$$

Notice that for $m, n \geq 0$ one has $\left\|\left(\varphi \circ \sigma^{n}\right)^{*} \circ \sigma^{m}-\left(\varphi \circ \sigma^{m+n}\right)^{*}\right\| \leq \operatorname{var}_{n} \tilde{\varphi}$. Hence

$$
\left|\int\left(\varphi \circ \sigma^{n}\right)^{*} d \mu-\int\left(\varphi \circ \sigma^{n+m}\right)^{*} d \mu\right|=\left|\int_{\leq}\left(\varphi \circ \sigma^{n}\right)^{*} \circ \sigma^{m} d \mu-\int\left(\varphi \circ \sigma^{n+m}\right)^{*} d \mu\right|
$$

tends to zero as $n \rightarrow \infty$ (because $\varphi$ is continuous). This proves that the latter is a Cauchy sequence and that the limit $\int \tilde{\varphi} d \tilde{\mu}=\lim _{n \rightarrow \infty} \int\left(\varphi \circ \sigma^{n}\right)^{*} d \mu$ exists. It is straightforward to check that $\tilde{\mu} \in C^{0}\left(\Sigma_{\mathbf{R}}, \mathbb{R}\right)^{*}$. By the Riesz Representation Theorem we see that $\tilde{\mu}$ defines a probability measures on $\Sigma_{\mathbf{R}}$. Note that $\int \varphi \circ \sigma d \tilde{\mu}=$ $\lim _{n \rightarrow \infty} \int\left(\varphi \circ \sigma^{n+1}\right)^{*} d \mu=\int \tilde{\varphi} d \tilde{\mu}$ for every continuous $\varphi$, proving that $\tilde{\mu}$ is $\sigma$-invariant. Also $\int \tilde{\varphi} d \tilde{\mu}=\int \varphi d \mu$ for $\varphi \in C^{0}\left(\Sigma_{\mathbf{R}}^{+}, \mathbb{R}\right)$.

Note that if $\psi=\varphi+u \circ \sigma-u$, then $M(\varphi, \sigma)=M(\psi, \sigma)$ and the maximizing measures for $\varphi$ and $\psi$ are the same. Hence, by Lemma 3.3 the pre-image $\Xi^{-1}\left(O^{+}\right)$is an open and dense subset of $C^{\alpha}\left(\Sigma_{\mathbf{R}}, \mathbb{R}\right)$, and for every $\varphi \in \Xi^{-1}\left(O^{+}\right)$there exists a single $\varphi$-maximizing measure and it is supported on a periodic orbit.

\section{ERGODIC OPTIMIZATION FOR SUSPENSION SEMIFLOWS AND APPLICATIONS}

This section contains some of the key reduction arguments explored in the paper. Throughout this section let $(N, d)$ be a compact metric space, let $f: N \rightarrow N$ be a continuous map and $r: N \rightarrow \mathbb{R}_{+}$be a continuous roof function bounded away from zero. Let $\left(X^{t}\right)_{t \in \mathbb{R}}$ be the suspension flow over $f$ with height function $r$. Given $\mu \in \mathcal{M}_{1}\left(N^{r},\left(X^{t}\right)_{t}\right)$ denote by $\bar{\mu} \in \mathcal{M}_{1}(N, f)$ the $f$-invariant probability measure induced by $\mu$, and recall that $\mu=\frac{\bar{\mu} \times L e b}{\int_{N} r d \bar{\mu}}$. 


\subsection{Reduction to the ergodic optimization of the Poincaré map.}

Lemma 4.1. Fix $\alpha>0$. If $r$ is a continuous (resp. $\alpha$-Hölder) roof function and $\Phi: N^{r} \rightarrow \mathbb{R}$ is a continuous (resp. $\alpha$-Hölder) observable then the induced observable $\varphi: N \rightarrow \mathbb{R}$ defined by $\varphi(x)=\int_{0}^{r(x)} \Phi(x, s) d s$, is a continuous (resp. $\alpha$-Hölder) observable and $\int_{N^{r}} \Phi d \mu=\frac{\int_{N} \varphi d \bar{\mu}}{\int_{N} r d \bar{\mu}}$.

Proof. Since $\mu=\frac{\bar{\mu} \times L e b}{\int_{N} r d \bar{\mu}}$ then

$$
\begin{aligned}
\int_{N^{r}} \Phi d \mu & =\int \Phi \circ \chi_{N^{r}} d \mu=\frac{1}{\int_{N} r d \bar{\mu}} \int_{N \times \mathbb{R}_{+}} \Phi \circ \chi_{N^{r}}(x, s) d \bar{\mu} \times L e b \\
& =\frac{1}{\int_{N} r d \bar{\mu}} \int_{N} \int_{0}^{r(x)} \Phi(x, s) d s d \bar{\mu}=\frac{\int_{N} \varphi d \bar{\mu}}{\int_{N} r d \bar{\mu}} .
\end{aligned}
$$

It remains to establish the regularity of the induced observable. Take $x, y \in N$ with $r(x) \geq r(y)$ (the case $r(x) \leq r(y)$ is analogous). Using that $\Phi$ and $r$ are continuous, we have

$$
\begin{aligned}
|\varphi(x)-\varphi(y)| & =\left|\int_{0}^{r(x)} \Phi(x, s) d s-\int_{0}^{r(y)} \Phi(y, s) d s\right| \\
& \leq \int_{0}^{r(y)}|\Phi(x, s)-\Phi(y, s)| d s+\int_{r(y)}^{r(x)} \Phi(x, s) d s \\
& \leq \sup r \cdot \sup _{s \in(0, r(y))}|\Phi(x, s)-\Phi(y, s)|+\sup |\Phi| \cdot|r(x)-r(y)|
\end{aligned}
$$

which ensures the continuity of $\varphi$. If, in addition, $\Phi$ and $r$ are $\alpha$-Hölder continuous then one can use proceed to bound (4.1) and obtain

$$
|\varphi(x)-\varphi(y)| \leq b \cdot \sup _{s \in(0, r(y))} d_{N^{r}}((x, s),(y, s))^{\alpha}+\sup |\Phi| L d_{N}(x, y)^{\alpha}
$$

for some positive constants $L$ and $b$. It follows from Proposition 2.14, inequality (4.2) and the relation of $d_{N^{r}}$ with the pseudo metric $d_{\pi}$ expressed in (2.5) that

$$
\begin{aligned}
|\varphi(x)-\varphi(y)| & \leq \sup |\Phi| L d_{N}(x, y)^{\alpha}+b c \sup _{s \in(0, r(y))} d_{\pi}((x, s),(y, s))^{\alpha} \\
& \leq[\sup |\Phi| \cdot L+b c] d_{N}(x, y)^{\alpha} .
\end{aligned}
$$

This proves the regularity of the induced observable in the Hölder category and finishes the proof of the lemma.

The next result allow us to reduce the ergodic optimization of suspension semiflows to the ergodic optimization of the continuous base dynamics, with respect to the induced observables.

Lemma 4.2. Let $\left(X^{t}\right)_{t}: N^{r} \rightarrow N^{r}$ be a suspension flow over a continuous map $f: N \rightarrow N$ on a compact metric space $N$ with continuous height function $r: N \rightarrow \mathbb{R}_{+}$. Let $\Phi: N^{r} \rightarrow \mathbb{R}$ be continuous and $\varphi: N \rightarrow \mathbb{R}$ be given by $\varphi(x)=\int_{0}^{r(x)} \Phi(x, s) d s$. The following are equivalent:

(1) $\mu$ is a maximizing measure for $\left(X^{t}\right)_{t}$ with respect to $\Phi$;

(2) $\bar{\mu}$ is a maximizing measure for $f$ with respect to $\tilde{\varphi}:=\varphi-M\left(\Phi,\left(X^{t}\right)_{t}\right) r$.

In both cases, $M(\tilde{\varphi}, f)=0$. 
Proof. First, Lemma 4.1 ensures that

$$
M\left(\Phi,\left(X^{t}\right)_{t}\right)=\max \left\{\int \Phi d v: v \in \mathcal{M}_{1}\left(N^{r},\left(X^{t}\right)_{t}\right)\right\}=\max \left\{\frac{\int_{N} \varphi d \bar{v}}{\int_{N} r d \bar{v}}: \bar{v} \in \mathcal{M}_{1}(N, f)\right\} .
$$

Therefore $M\left(\Phi,\left(X^{t}\right)_{t}\right) \geq \frac{\int_{N} \varphi d \bar{v}}{\int_{N} r d \bar{v}}$ for all $\bar{v} \in \mathcal{M}_{1}(N, f)$ and, consequently,

$$
\max _{\bar{v} \in \mathcal{M}_{1}(N, f)} \int_{N}\left(\varphi-M\left(\Phi,\left(X^{t}\right)_{t}\right) r\right) d \bar{v} \leq 0 .
$$

Moreover, if $\mu$ is a maximizing measure for $\left(X^{t}\right)_{t}$ with respect to $\Phi$ and $\bar{\mu}$ is as before then

$$
\begin{aligned}
\int_{N}\left(\varphi-M\left(\Phi,\left(X^{t}\right)_{t}\right) r\right) d \bar{\mu} & =\int_{N} \varphi d \bar{\mu}-M\left(\Phi,\left(X^{t}\right)_{t}\right) \int_{N} r d \bar{\mu} \\
& =\int_{N} \varphi d \bar{\mu}-\int_{N^{r}} \Phi d \mu \int_{N} r d \bar{\mu}=0 .
\end{aligned}
$$

Since zero is the maximum possible value for $\int_{N}\left(\varphi-M\left(\Phi,\left(X^{t}\right)_{t}\right) r\right) d \bar{\mu}$ (recall (4.3)), $\bar{\mu}$ is a maximizing measure for $\tilde{\varphi}=\varphi-M\left(\Phi,\left(X^{t}\right)_{t}\right) r$ with respect to $f$ and $M(\tilde{\varphi}, f)=0$.

Conversely, suppose that $\bar{\mu}$ is a maximizing measure for $\tilde{\varphi}:=\varphi-M\left(\Phi,\left(X^{t}\right)_{t}\right) r$ with respect to $f$. We claim that $M(\tilde{\varphi}, f)=0$. Suppose by contradiction that $M(\tilde{\varphi}, f)=\max _{\bar{v} \in \mathcal{M}_{1}(N, f)} \int_{N}\left(\varphi-M\left(\Phi,\left(X^{t}\right)_{t}\right) r\right) d \bar{v}<0$. In this case,

$$
\frac{M(\tilde{\varphi}, f)}{\int_{N} r d \bar{v}} \leq \frac{M(\tilde{\varphi}, f)}{\|r\|_{\infty}}<0
$$

since, for any $\bar{v} \in \mathcal{M}_{1}(N, f), \int_{N} r d \bar{v} \leq\|r\|_{\infty}$. Consequently $\int_{N}\left(\varphi-M\left(\Phi,\left(X^{t}\right)_{t}\right) r\right) d \bar{v} \leq M(\tilde{\varphi}, f)<0$ implies that

$$
\frac{\int_{N} \varphi d \bar{\nu}}{\int_{N} r d \bar{v}}-\frac{M\left(\Phi,\left(X^{t}\right)_{t}\right) \int_{N} r d \bar{v}}{\int_{N} r d \bar{v}} \leq \frac{M(\tilde{\varphi}, f)}{\int_{N} r d \bar{v}}<0
$$

and so $\int_{N^{r}} \Phi d v-M\left(\Phi,\left(X^{t}\right)_{t}\right) \leq \frac{M(\tilde{\varphi}, f)}{\|r\|_{\infty}}<0$. Therefore there is $a>0$ such that $\int_{N^{r}} \Phi d v-M\left(\Phi,\left(X^{t}\right)_{t}\right)<-a$ for all $v \in \mathcal{M}_{1}\left(N^{r},\left(X^{t}\right)_{t}\right)$ and taking the maximum over $v$ we conclude that $\max _{v \in \mathcal{M}_{1}\left(N^{r},\left(X^{t}\right)_{t}\right)} \int_{N^{r}} \Phi d v-M\left(\Phi,\left(X^{t}\right)_{t}\right)<$ $-a<0$, leading to a contradiction and proving the claim. Now, if $\bar{\mu}$ is a maximizing measure with respect to $\tilde{\varphi}$ then $\int_{N}\left(\varphi-M\left(\Phi,\left(X^{t}\right)_{t}\right) r\right) d \bar{\mu}=0$ and, by Lemma 4.1, $M\left(\Phi,\left(X^{t}\right)_{t}\right)=\frac{\int_{N} \varphi d \bar{\mu}}{\int_{N} r d \bar{\mu}}=\int_{N^{r}} \Phi d \mu$, which proves that $\mu$ is a maximizing measure for $\Phi$ with respect to $\left(X^{t}\right)_{t}$.

4.2. Ergodic optimization: from discrete-time to continuous time. In Subsection 4.1 we proved that one can relate the ergodic optimization for semiflows with the one for the Poincaré return maps. However such a relation, expressed by Lemma 4.2 could a priori relate a topologically large set of observables on the suspension space $N^{r}$ with a meager set of observables on $N$. In its essence the next lemmas will allow us to see that this is not the case and provides a correspondence between maximizing measures for potentials on the Poincaré map and maximizing measures for suspension semiflows.

Lemma 4.3. Fix $\alpha \geq 0$. The map $\mathfrak{F}: C^{\alpha}\left(N^{r}, \mathbb{R}\right) \rightarrow C^{\alpha}(N, \mathbb{R})$ given by

$$
\mathfrak{F}(\Phi)=\int_{0}^{r(x)} \Phi(x, s) d s
$$

is a submersion. 
Proof. As $\mathfrak{F}$ is linear (thus $D_{\Phi} \mathfrak{F}(H)=\mathfrak{F}(H)$ for $H \in C^{\alpha}\left(N^{r}, \mathbb{R}\right)$ ), in order to prove that $\mathfrak{F}$ is a submersion we need only prove that it is surjective. Indeed, for any $\varphi \in C^{\alpha}(N, \mathbb{R})$ the observable $\Phi(x, t)=\frac{\varphi(x)}{r(x)}$ belongs to $C^{\alpha}\left(N^{r}, \mathbb{R}\right)$ (because $r(x)>0$ for every $\left.x \in N\right)$ and $\mathfrak{F}(\Phi)=\int_{0}^{r(x)} \Phi(x, s) d s=\int_{0}^{r(x)} \frac{\varphi(x)}{r(x)} d s=\varphi(x)$. Therefore $D_{\Phi} \mathfrak{F}$ is surjective and $\mathfrak{F}$ is a submersion.

In view of Lemma 4.3 we can expect the ergodic optimization for discrete-time dynamics to be lifted to the context of suspension semiflows. On the one hand, the pre-image of an open (resp. dense) set by $\mathfrak{F}$ is open (resp. dense). However, on the other hand, the image of $\mathfrak{F}$ does not restrict to the set of observables with maximum zero, a condition that is crucial in the relation between ergodic optimization of the flows and the Poincaré maps (recall item (2) in Lemma 4.2).

In order to overcome this issue we consider a further decomposition on the vector spaces of observables. Given $\alpha \geq 0$ we say that a property (P) on the maximizing measures associated of elements in $C^{\alpha}(N, \mathbb{R})$ is invariant under translation by constants whenever the following holds: the maximizing measures of $\varphi \in$ $C^{\alpha}(N, \mathbb{R})$ satisfy $(\mathrm{P})$ if and only if the maximizing measures of $\varphi+k \in C^{\alpha}(N, \mathbb{R})$ satisfy $(\mathrm{P})$ for every $k \in \mathbb{R}$. For instance, the property $(\mathrm{P})$ of having a unique maximizing measure is clearly invariant under translation by constants. The following is the main result of this section.

Proposition 4.4. Let $\alpha \geq 0$ and let $(P)$ be a property on the maximizing measures of the elements of $C^{\alpha}(N, \mathbb{R})$ that is invariant under translation by constants. Let $\mathcal{P}$ be the space of all $\varphi \in C^{\alpha}(N, \mathbb{R})$ so that all $\varphi$-maximizing measures satisfy $(P)$. Then the following hold:

(i) if $\mathcal{P}$ is dense in $C^{\alpha}(N, \mathbb{R})$ then the set of observables $\Phi \in C^{\alpha}\left(N^{r}, \mathbb{R}\right)$ so that all $\Phi$-maximizing measures satisfy $(P)$ is dense in $C^{\alpha}\left(N^{r}, \mathbb{R}\right)$; and

(ii) if $\mathcal{P}$ is open in $C^{\alpha}(N, \mathbb{R})$ then the set of observables $\Phi \in C^{\alpha}\left(N^{r}, \mathbb{R}\right)$ so that all $\Phi$-maximizing measures satisfy $(P)$ is open in $C^{\alpha}\left(N^{r}, \mathbb{R}\right)$.

Proof. Let $\alpha \geq 0$, let (P) be a property as above. One can write

$$
C^{\alpha}(N, \mathbb{R})=\bigcup_{k \in \mathbb{R}} C_{k}, \quad \text { where } \quad C_{k}:=\left\{\psi \in C^{\alpha}(N, \mathbb{R}): M(\psi, f)=k\right\}
$$

is a level set of maximization for every $k \in \mathbb{R}$. Consider the map $\pi_{0}: C^{\alpha}(N, \mathbb{R}) \rightarrow C_{0}$ given by $\pi_{0}(\varphi)=$ $\varphi-M(\varphi, \sigma)$. Our assumption implies that all $\varphi$-maximizing measures satisfy property (P) if and only if all $\pi_{0}(\varphi)$-maximizing measures satisfy property $(\mathrm{P})$. While it is unclear if the map $\pi_{0}$ is a submersion (as is would require differentiability of the function $\varphi \mapsto M(\varphi, \sigma)$ ), the first step in the proof (Claims 1 and 2 below) consists of proving that the space of observables whose maximizing measures satisfy $(\mathrm{P})$ have the same structure along the level sets defined in (4.5).

Claim 1. If $O \subset C^{\alpha}(N, \mathbb{R})$ is open then $\pi_{0}(O)$ is open in $C_{0}$.

Claim 2. If $O \subset C^{\alpha}(N, \mathbb{R})$ is dense then $\pi_{0}(O)$ is dense in $C_{0}$.

Proof of Claim 1. Take any $\varphi_{1} \in \pi_{0}(O)$. We will show that $\varphi_{1}$ is an interior point of $\pi_{0}(O)$ in $C_{0}$. There exists $\psi_{1} \in O$ such that $\varphi_{1}=\psi_{1}-M\left(\psi_{1}, \sigma\right)$. Denote $k_{1}=M\left(\psi_{1}, \sigma\right)$ and consider the set $C_{k_{1}}$, as in (4.5). Since $O$ is open in $C^{\alpha}(N, \mathbb{R}), O \cap C_{k_{1}}$ is open in $C_{k_{1}}$, so there is $\varepsilon_{1}>0$ such that $B\left(\psi_{1}, \varepsilon_{1}\right) \cap C_{k_{1}} \subset O \cap C_{k_{1}}$, where $B\left(\psi_{1}, \varepsilon_{1}\right)$ is the open ball in $C^{\alpha}(N, \mathbb{R})$ with center in $\psi_{1}$ and radius $\varepsilon_{1}$. For any $\varphi_{2} \in B\left(\varphi_{1}, \varepsilon_{1}\right) \cap C_{0}$ define $\psi_{2}:=\varphi_{2}+k_{1}$. Since $M\left(\varphi_{2}, \sigma\right)=0$, we have that $M\left(\psi_{2}, \sigma\right)=k_{1}$, hence $\psi_{2} \in C_{k_{1}}$ and $\left\|\psi_{1}-\psi_{2}\right\|=\left\|\psi_{1}-\varphi_{2}-k_{1}\right\|=\left\|\varphi_{1}-\varphi_{2}\right\| \leq$ $\varepsilon_{1}$, so $\psi_{2} \in B\left(\psi_{1}, \varepsilon_{1}\right)$. Therefore $\psi_{2} \in C_{k_{1}}$ and $\varphi_{2} \in \pi_{0}(O)$. Since $\varphi_{2}$ was taken arbitrarily, we have that $B\left(\varphi_{1}, \varepsilon_{1}\right) \cap C_{0} \subset \pi_{0}(O)$, which means that $\varphi_{1}$ is an interior point of $\pi_{0}(O)$ in $C_{0}$. Therefore $\pi_{0}(O)$ is an open subset of $C_{0}$, which proves the claim. 
Proof of Claim 2. In order to prove that $\pi_{0}(O)$ is dense, for any $\varphi_{3} \in C_{0} \backslash \pi_{0}(O)$ we show that $\varphi_{3}$ is a accumulation point for $\pi_{0}(O)$ in $C_{0}$. Since $O$ is dense in $C^{\alpha}(N, \mathbb{R})$, there is $\left\{\psi_{n}\right\}_{n} \subset O$ such that $\psi_{n} \rightarrow \varphi_{3}$ as $n \rightarrow \infty$. Since $C^{\alpha}(N, \mathbb{R}) \ni \varphi \mapsto M(\varphi, \sigma)$ is continuous, we have that $\pi_{0}$ is also continuous, so $\pi_{0}\left(\psi_{n}\right) \rightarrow \pi_{0}\left(\varphi_{3}\right)=\varphi_{3}$ as $n \rightarrow \infty$ (cf. Figure 1). Therefore $\varphi_{3}$ is a accumulation point of elements in $\pi_{0}(O)$, which means that $\pi_{0}(O)$ is

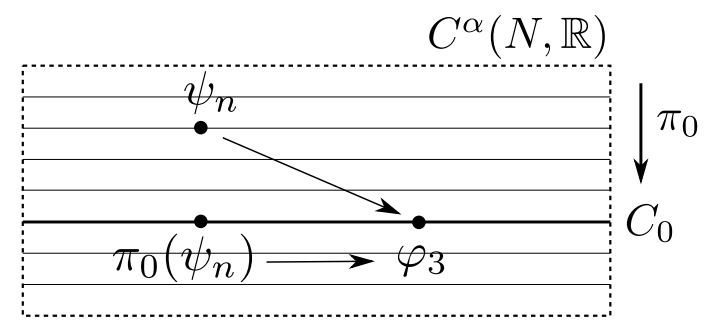

FigURE 1. $\psi_{n} \rightarrow \varphi_{3} \Rightarrow \pi_{0}\left(\psi_{n}\right) \rightarrow \varphi_{3}$.

dense in $C_{0}$. This proves the claim.

The second step in the proof of Proposition 4.4 is to ensure that some properties on the space of observables lift from the context of maps to the context of semiflows. Let $\mathfrak{F}$ be given by Lemma 4.3. We have that the set $C_{0}^{r}=\left\{\Phi \in C^{\alpha}\left(N^{r}, \mathbb{R}\right): M\left(\Phi,\left(X^{t}\right)_{t}\right)=0\right\}$ is mapped by $\mathfrak{F}$ onto $C_{0}$ by $\mathfrak{F}$, that is, $\mathfrak{F}\left(C_{0}^{r}\right)=C_{0}$. In fact, take $\Phi \in C_{0}^{r}$, that is, $M\left(\Phi,\left(X^{t}\right)_{t}\right)=0$. Writing $\tilde{\varphi}:=\varphi-M\left(\Phi,\left(X^{t}\right)_{t}\right) r$, where $\varphi=\mathfrak{F}(\Phi)$, we have that $\tilde{\varphi}=\varphi$ and $M(\varphi, \sigma)=0$, by Lemma 4.2 .

Now take $\varphi \in C_{0}$, meaning that $M(\varphi, \sigma)=0$. Since $\mathfrak{F}$ is surjective, there exists $\Phi \in C^{\alpha}\left(\Sigma_{\mathbf{R}}^{r}, \mathbb{R}\right)$ such that $\varphi=\int_{0}^{r(x)} \Phi(x, s) d s$. We claim that $M\left(\Phi,\left(X^{t}\right)_{t}\right)=0$. Let $\mu_{\varphi}$ be $\sigma$-invariant such that $\int \varphi d \mu_{\varphi}=M(\varphi, \sigma)=0$ and let $\mu_{\Phi}$ be $\left(X^{t}\right)_{t}$-invariant such that $\int \Phi d \mu_{\Phi}=M\left(\Phi,\left(X^{t}\right)_{t}\right)$. If $\bar{\mu}_{\Phi}$ is the unique $\sigma$-invariant probability so that $\mu_{\Phi}=\frac{\bar{\mu}_{\Phi} \times L e b}{\int r d \bar{\mu}_{\Phi}}$, Lemmas 4.1 and 4.2 ensure that

$$
M\left(\Phi,\left(X^{t}\right)_{t}\right)=\int \Phi d \mu_{\Phi}=\frac{\int \varphi d \bar{\mu}_{\Phi}}{\int r d \bar{\mu}_{\Phi}}
$$

that $\int\left[\varphi-M\left(\Phi,\left(X^{t}\right)_{t}\right) r\right] d \bar{\mu}_{\Phi}=0$. Now, equation (4.6) together with the fact that $\int \varphi d \bar{\mu}_{\Phi} \leq M(\varphi, \sigma)=0$ implies $M\left(\Phi,\left(X^{t}\right)_{t}\right) \leq 0$. Thus $\varphi-M\left(\Phi,\left(X^{t}\right)_{t}\right) r \geq \varphi$ and

$$
0=\int\left[\varphi-M\left(\Phi,\left(X^{t}\right)_{t}\right) r\right] d \bar{\mu}_{\Phi} \geq \int\left[\varphi-M\left(\Phi,\left(X^{t}\right)_{t}\right) r\right] d \mu_{\varphi} \geq \int \varphi d \mu_{\varphi}=0 .
$$

This ensures that $\int\left[\varphi-M\left(\Phi,\left(X^{t}\right)_{t}\right) r\right] d \mu_{\varphi}=0$ and, consequently,

$$
M\left(\Phi,\left(X^{t}\right)_{t}\right)=\frac{\int \varphi d \mu_{\varphi}}{\int r d \mu_{\varphi}}=0 .
$$

This shows that $\mathfrak{F}\left(C_{0}^{r}\right) \supset C_{0}$. Moreover, since $\mathfrak{F}$ is a submersion, Claims 1 and 2 ensure that the pre-image $\mathfrak{F}^{-1}\left(\pi_{0}(O)\right)$ is an open (resp. dense) subset of $C_{0}^{r}$ whenever $O$ is an open (resp. dense) subset of $C^{\alpha}(N, \mathbb{R})$. Now consider the projection $\Pi_{0}: C^{\alpha}\left(N^{r}, \mathbb{R}\right) \rightarrow C_{0}^{r}$ defined by $\Phi \mapsto \Phi-M\left(\Phi,\left(X^{t}\right)_{t}\right)$. Since the maximizing measure of every element in $\mathfrak{F}^{-1}\left(\pi_{0}(O)\right)$ satisfies property $(\mathrm{P})$ then we are left to prove the following:

Claim 3. If $O \subset C^{\alpha}(N, \mathbb{R})$ is open (resp. dense) then $\Pi_{0}^{-1}\left(\mathfrak{F}^{-1}\left(\pi_{0}(O)\right)\right)$ is an open (resp. dense) subset of $C^{\alpha}\left(N^{r}, \mathbb{R}\right)$. 
Proof. In order to prove that $\Pi_{0}^{-1}\left(\mathfrak{F}^{-1}\left(\pi_{0}(O)\right)\right)$ is open we prove that any $\Phi \in \Pi_{0}^{-1}\left(\mathfrak{F}^{-1}\left(\pi_{0}(O)\right)\right)$ in an interior point. Let $\Phi_{0}:=\Pi_{0}(\Phi)=\Phi-M\left(\Phi,\left(X^{t}\right)_{t}\right) \in \mathfrak{F}^{-1}\left(\pi_{0}(O)\right)$. Since $\mathfrak{F}^{-1}\left(\pi_{0}(O)\right)$ is open in $C_{0}^{r}$, there exists $\varepsilon>0$ such that if $\Upsilon \in C_{0}^{r}$ and $\left\|\Phi_{0}-\Upsilon\right\|<\varepsilon$ then $\Upsilon \in \mathfrak{F}^{-1}\left(\pi_{0}(O)\right)$. On the other hand, since $\Phi \mapsto M\left(\Phi,\left(X^{t}\right)_{t}\right)$ is a continuous map, there is $0<\delta<\varepsilon / 2$ such that if $\|\Phi-\Psi\|<\delta$, then $\left|M\left(\Phi,\left(X^{t}\right)_{t}\right)-M\left(\Psi,\left(X^{t}\right)_{t}\right)\right|<\frac{\varepsilon}{2}$. So if $\Psi \in C^{\alpha}\left(N^{r}, \mathbb{R}\right)$ satisfies $\|\Phi-\Psi\|<\delta$ then

$$
\begin{aligned}
\left\|\Phi_{0}-\Psi_{0}\right\| & =\left\|\Phi-M\left(\Phi,\left(X^{t}\right)_{t}\right)-\Psi+M\left(\Psi,\left(X^{t}\right)_{t}\right)\right\| \\
& \leq\|\Phi-\Psi\|+\left\|M\left(\Phi,\left(X^{t}\right)_{t}\right)-M\left(\Psi,\left(X^{t}\right)_{t}\right)\right\|<\delta+\frac{\varepsilon}{2}<\varepsilon
\end{aligned}
$$

which means that $\Psi_{0} \in C_{0}^{r}$. So $\Psi \in \Pi_{0}^{-1}\left(\mathfrak{F}^{-1}\left(\pi_{0}(O)\right)\right)$ and consequently $\Pi_{0}^{-1}\left(\mathfrak{F}^{-1}\left(\pi_{0}(O)\right)\right)$ is open. In order to show that $\Pi_{0}^{-1}\left(\mathfrak{F}^{-1}\left(\pi_{0}(O)\right)\right)$ is dense, we take any $\Psi \in C^{\alpha}\left(N^{r}, \mathbb{R}\right) \backslash \Pi_{0}^{-1}\left(\mathfrak{F}^{-1}\left(\pi_{0}(O)\right)\right)$ and show that $\Psi$ is a accumulation point of elements in $\Pi_{0}^{-1}\left(\mathfrak{F}^{-1}\left(\pi_{0}(O)\right)\right)$. Since $\mathfrak{F}^{-1}\left(\pi_{0}(O)\right)$ is dense in $C_{0}^{r}$, for any $\varepsilon>0$ there is $\Upsilon \in \mathfrak{F}^{-1}\left(O_{0}\right)$ such that $\left\|\Psi_{0}-\Upsilon\right\|<\varepsilon$. Taking $\Phi=\Upsilon+M\left(\Psi,\left(X^{t}\right)_{t}\right)$, note that $M\left(\Phi,\left(X^{t}\right)_{t}\right)=M\left(\Psi,\left(X^{t}\right)_{t}\right)$. Moreover $\Phi \in \Pi_{0}^{-1}\left(\mathfrak{F}^{-1}\left(\pi_{0}(O)\right)\right)$, because $M\left(\Phi,\left(X^{t}\right)_{t}\right)=M\left(\Psi,\left(X^{t}\right)_{t}\right)$ and

$$
\Phi_{0}=\Phi-M\left(\Phi,\left(X^{t}\right)_{t}\right)=\Upsilon+M\left(\Psi,\left(X^{t}\right)_{t}\right)-M\left(\Psi,\left(X^{t}\right)_{t}\right)=\Upsilon \in \widetilde{F}^{-1}\left(\pi_{0}(O)\right) .
$$

We also have $\|\Psi-\Phi\|=\left\|\Psi-\Upsilon-M\left(\Psi,\left(X^{t}\right)_{t}\right)\right\|=\left\|\Psi_{0}-\Upsilon\right\|<\varepsilon$. Since $\varepsilon$ was arbitrary the density of $\Pi_{0}^{-1}\left(\mathfrak{F}^{-1}\left(\pi_{0}(O)\right)\right)$ follows. This finishes the proof of the claim.

The proof of the proposition is now complete.

4.3. Suspension flows over subshifts of finite type. The following result summarizes combines Proposition 4.4 together with the results on ergodic optimization for subshifts of finite type in Subsection 3.2.

Theorem 4.5. Let $\sigma: \Sigma_{\mathbf{R}} \rightarrow \Sigma_{\mathbf{R}}$ be a transitive two-sided subshift of finite type, let $r: \Sigma_{\mathbf{R}} \rightarrow \mathbb{R}$ be a continuous roof function bounded away from zero and let $\left(X^{t}\right)_{t \in \mathbb{R}}$ be the suspension flow over $\sigma$ with height function $r$. Then the following hold:

(1) exists an open and dense subset $O \subset C^{0}\left(\Sigma_{\mathbf{R}}, \mathbb{R}\right)$ so that every $\Phi \in O$ has a unique $\left(X^{t}\right)_{t}$-maximizing measure with respect to $\Phi$, it has zero entropy and full support;

(2) exists a dense set $\mathcal{D} \subset C^{0}\left(\Sigma_{\mathbf{R}}, \mathbb{R}\right)$ so that every $\Phi \in \mathcal{D}$ has uncountably many ergodic $\Phi$-maximizing measures;

(3) if, in addition, $r$ is Hölder continuous then exists an open and dense set $\mathcal{R}_{r} \subset C^{\alpha}\left(\Sigma_{\mathbf{R}}^{r}, \mathbb{R}\right)$ of observables $\Phi: \Sigma_{\mathbf{R}}^{r} \rightarrow \mathbb{R}$ so that every $\Phi \in \mathcal{R}_{r}$ has a unique $\left(X^{t}\right)_{t}$-maximizing measure with respect to $\Phi$, and it is supported on a periodic orbit;

Proof. On the one hand, by Proposition 3.5, there exists an open and dense set $O \subset C^{\alpha}\left(\Sigma_{\mathbf{R}}, \mathbb{R}\right)$ such that every $\varphi \in O$ has a unique $\varphi$-maximizing measure $\bar{\mu}$ and it is supported on a periodic orbit. On the other hand, since $\sigma$ is a transitive subshift of finite type then it satisfies the gluing orbit property [7]. Thus Theorem 2.5 ensures that there exists a dense $G_{\delta}$ subset $\mathcal{Z} \subset C^{0}\left(\Sigma_{\mathbf{R}}, \mathbb{R}\right)$ so that every $\varphi \in \mathcal{Z}$ has a unique $\varphi$-maximizing measure, with zero entropy and full support. Finally, the results in Subsection 3.2 to obtain conclusions for bilateral subshifts of finite type together with Theorem 2.8 ensure that exists a dense subset $\mathcal{D} \subset C^{0}\left(\Sigma_{R}, \mathbb{R}\right)$ such that for every $\varphi \in \mathcal{D}$ there exist uncountably many ergodic $\varphi$-maximizing measures with full support and positive entropy. Hence, the corollary is a direct consequence of Theorem 4.4. 
4.4. Ergodic optimization on hyperbolic sets. Let $\Lambda \subset M$ be a horseshoe for the flow $\left(X^{t}\right)_{t \in \mathbb{R}}$. Since hyperbolic flows admit Markov partitions, these are modeled by suspension flows [12, 38]. Then Theorem A follows from corresponding result for suspension flows (cf. Theorem 4.5). Indeed, there is a subshift of finite type $\sigma_{\mathbf{R}}: \Sigma_{\mathbf{R}} \rightarrow \Sigma_{\mathbf{R}}$, a positive $r \in C^{\alpha}\left(\Sigma_{\mathbf{R}}, \mathbb{R}\right)$ and a Hölder continuous homeomorphism $\pi: \Sigma_{\mathbf{R}}^{r} \rightarrow \Lambda$ so that

$$
\pi \circ Y^{t}=X^{t} \circ \pi \quad \text { for every } t \in \mathbb{R},
$$

where $\Sigma_{\mathbf{R}}^{r}$ is a quotient as in Subsection 2.4 and $\left(Y^{t}\right)_{t}: \Sigma_{\mathbf{R}}^{r} \rightarrow \Sigma_{\mathbf{R}}^{r}$ is the suspension flow over $\sigma_{\mathbf{R}}$ with height function $r$. If $\Lambda$ is a horseshoe then $\pi: \Sigma_{\mathbf{R}}^{r} \rightarrow \Lambda$ is one-to-one. So given an observable $\Phi \in C^{\alpha}(\Lambda, \mathbb{R})$ one can induce an observable $\Phi^{*} \in C^{\alpha}\left(\Sigma_{\mathbf{R}}^{r}, \mathbb{R}\right)$ by doing $\Phi^{*}=\Phi \circ \pi$ and the map $\Theta: C^{\alpha}(\Lambda, \mathbb{R}) \rightarrow C^{\alpha}\left(\Sigma_{\mathbf{R}}^{r}, \mathbb{R}\right)$ defined by $\Theta(\Phi)=\Phi \circ \pi$ is one-to-one. Theorem A is now a direct consequence of Theorem 4.5.

\section{Applichtion to LoREnZ-LiKe atTractors}

In this section we use Theorem A to prove Theorem B by an approximation method. The proof of the latter also relies on auxiliary results on the structure of invariant subsets of Lorenz attractors and the space of invariant probability measures for wild Lorenz attractors.

5.1. Proper subsets and the space of invariant probabilities. Let $N$ be a three-dimensional closed Riemannian manifold, $\Lambda \subset N$ be a geometric Lorenz attractor for the $C^{1}$-flow $\left(X^{t}\right)_{t}$ generated by a $C^{1}$-vector field $X$ and let $\mathcal{W} \supset \Lambda$ denote an attracting region. Hence $\Lambda=\bigcap_{t \geq 0} X^{-t}(\mathcal{W})$. For every open neighborhood $\mathcal{U}$ of $\operatorname{Sing}(X)$ and every $\varphi \in C^{0}(N, \mathbb{R})$, and let the maximal invariant set $\Lambda_{\mathcal{U}}$ in $\mathcal{W} \backslash \mathcal{U}$ and the constrained ergodic optimization maximum be defined by

$$
\Lambda_{\mathcal{U}}:=\bigcap_{t \geq 0} X^{-t}(\mathcal{W} \backslash \mathcal{U}) \quad \text { and } \quad M_{\mathcal{U}}(\varphi)=\max _{\substack{\mu\left(\Lambda_{\mathcal{U}}\right)=1 \\ \mu \text { ergodic }}}\left\{\int \varphi d \mu\right\}
$$

respectively. The set $\Lambda_{\mathcal{U}}$ is a compact $\left(X^{t}\right)_{t}$-invariant singular-hyperbolic set without singularities, hence it is a uniformly hyperbolic set (cf. [2, Proposition 6.2]). In the case of more general singular-hyperbolic attractors $a$ priori the set $\Lambda_{\mathcal{U}}$ could be non-transitive. We prove this is not the case for geometric Lorenz attractors.

Lemma 5.1. Assume that $\Lambda$ is a geometric Lorenz attractor for the flow $\left(X^{t}\right)_{t}$. If $\mathcal{U}$ is any open neighborhood of $\sigma$ then the invariant set $\Lambda_{\mathcal{U}}$ defined by (5.1) is transitive.

Proof. Let $\Sigma$ denote the global cross-section to the flow $\left(X^{t}\right)_{t}$. Observe that it is enough to prove that the Poincaré return map $P$ defined in (2.2) is transitive on $\Lambda \mathcal{U} \cap \Sigma$. A direct proof of this single fact seems not easy. We prove transitivity by proving that $\Lambda_{\mathcal{U}} \cap \Sigma$ is a homoclinic class for $P$ (transitivity follows as a consequence of Birkhoff-Smale theorem).

Since periodic points are dense, in order to prove that $\Lambda \mathcal{U} \cap \Sigma$ is a homoclinic class for $P$ we claim that any two periodic points for $P$ are heteroclinically related. Let $p, q$ be any periodic points for $P$ (these are hyperbolic periodic points and we assume, without loss of generality, that $p, q$ are fixed points for $P)$. Let $W_{l o c}^{u}(p) \subset \Sigma$ denote the local Pesin unstable manifolds at $p$ and set $I=\pi^{s}\left(W_{l o c}^{u}(p)\right) \subset[-1,1] \backslash\{0\}$. Since one-dimensional Lorenz map $\alpha$ is locally eventually onto (recall Subsection 2.3), there exists $N \geq 1$ so that $\alpha^{N}(I)=(\alpha(-1), \alpha(1)$ ). This proves that $P^{N}\left(W_{l o c}^{u}(p)\right)$ crosses $\Sigma$, hence $W^{u}(p)$ intersects the stable manifold $W_{l o c}^{s}(q)$. Replacing the roles of $p$ and $q$ above we conclude that $p$ and $q$ are heteroclinically related. Therefore, $\Lambda \mathcal{U} \cap \Sigma$ is transitive. 
We proceed to describe the maximum value in ergodic optimization for flows. By the ergodic decomposition theorem the maximum is attained at ergodic measures and, consequently, the following equalities hold:

$$
\begin{aligned}
& M\left(\Phi,\left(X^{t}\right)_{t}\right)=\max _{\substack{\mu(\Lambda)=1 \\
\mu \text { ergodic }}}\left\{\int \Phi d \mu\right\} \\
& =\max \left\{\sup _{\substack{\mu \text { non-atomic } \\
\mu \text { ergodic }}}\left\{\int \Phi d \mu\right\}, \sup _{\substack{\mu \text { atomic } \\
\mu \text { ergodic }}}\left\{\int \Phi d \mu\right\}\right\} \\
& =\max \left\{\sup _{\substack{\text { supp } \mu=\Lambda \\
\mu \operatorname{ergodic}}}\left\{\int \Phi d \mu\right\}, \sup _{\substack{\text { supp } \mu \neq \Lambda \\
\mu \operatorname{ergodic}}}\left\{\int \Phi d \mu\right\}\right\} \\
& =\max \left\{\sup _{\substack{\operatorname{sing}(X) \cap \operatorname{supp} \mu \neq \emptyset \\
\mu \text { ergodic }}}\left\{\int \Phi d \mu\right\}, \sup _{\substack{\operatorname{Sing}(X) \cap \text { nupp } \\
\mu \text { ergodic }}}\left\{\int \Phi d \mu\right\}\right\} .
\end{aligned}
$$

In order to simplify notation we used $M\left(\Phi,\left(X^{t}\right)_{t}\right)$ instead of $M\left(\Phi,\left(X^{t}\right)_{t}, \Lambda\right)$ to denote the maximum value in ergodic optimization on the Lorenz attractor $\Lambda$. Using Theorem 2.1 we conclude that exactly one of the supremum terms in the right hand of each of the equations (5.2) - (5.4) is attained by some ergodic probability measure in the case of typical observables. Hence it makes natural to ask whether probabilities determined by periodic orbits are dense in the space of invariant measures for the Lorenz attractor. The next lemma gives a positive answer to this question.

Lemma 5.2. Assume that $\Lambda$ is a wild Lorenz attractor. The space of periodic probabilities is dense in the space $\mathcal{M}_{e}\left(\left(X_{t}\right)_{t}\right)$ of $\left(X_{t}\right)_{t}$-invariant probability measures on the attractor.

Proof. By the ergodic decomposition theorem (see e.g. [28]) it is enough to prove that every ergodic probability can be weak ${ }^{*}$ approximated by probabilities supported on periodic points. Since this is the case for every nonatomic ergodic probability (recall Remark 2.12) it remains to prove that the Dirac measure $\delta_{\sigma}$ is also weak* approximated by periodic probabilities.

Recall that the Lorenz attractor is modeled by a suspension flow. Moreover, changing the cross-section if necessary, one can assume that the return time function $\varrho$ is constant along the strong-stable leaves (see e.g. [3]) and

$$
\varrho(x)=-\frac{1}{\lambda_{1}} \log |x|+s(x)
$$

for some bounded function $s$. In particular, since $\alpha^{\prime}(x)=\gamma x^{1-\gamma}$ in a neighborhood of 0 (recall (2.3)) then there are constants $C_{1}, C_{2}>0$ so that

$$
C_{1} \log \left|\alpha^{\prime}(x)\right| \leq \varrho(x) \leq C_{2} \log \left|\alpha^{\prime}(x)\right| \text { for all } x \neq 0 .
$$

We claim that any sequence $\left(\mu_{n}\right)_{n}$ of $P$-invariant ergodic probability measures such that $\int \log \left|\alpha^{\prime}(x)\right| d \mu_{n} \rightarrow \infty$ as $n \rightarrow \infty$ converges in the weak* topology to the Dirac measure $\delta_{\sigma}$. Given $\varepsilon>0$ arbitrary let $V_{\varepsilon}$ be the ball of radius $\varepsilon$ around $\sigma$. Choose a local cross-section $\Sigma_{\varepsilon} \subset \Sigma$ so that the following hold:

(i) the piece of orbit $\left\{X^{t}(x): 0 \leq t \leq \varrho(x)\right\}$ of a point $x \in \Sigma_{\varepsilon}$ intersects $V_{\varepsilon}$, and

(ii) $\operatorname{Leb}\left(0 \leq t \leq \varrho(x): X^{t}(x) \in V_{\varepsilon}\right)>(1-\varepsilon) \varrho(x)$ for every $x \in \Sigma_{\varepsilon}$.

The construction of a local cross-section $\Sigma_{\varepsilon}$ satisfying (i) and (ii) is possible taking $\Sigma_{\varepsilon}$ as a small neighborhood of the stable manifold of $W^{s}(\sigma) \cap \Sigma$ and noting that, due to the continuous dependence of solutions of ordinary 
differential equations and that the return time function can be chosen arbitrary large for points close to the stable manifold, which ensures that these orbits spend as much proportion of time near the singularity as desired.

By construction $\Sigma_{\varepsilon}$ is a small neighborhood of the stable manifold of $W^{s}(\sigma) \cap \Sigma$, hence the roof function $\varrho$ is bounded by some constant $C_{\varepsilon}$ all points in $\Sigma \backslash \Sigma_{\varepsilon}$. Relation (5.6) implies that $\int_{\Sigma_{\varepsilon}} \varrho d \mu_{n} \rightarrow \infty$ as $n \rightarrow \infty$. For any $T>0$ let $N(x, T) \geq 1$ be the unique integer such that $\sum_{j=0}^{N(x, T)-1} \varrho\left(P^{j}(x)\right) \leq T<\sum_{j=0}^{N(x, T)} \varrho\left(P^{j}(x)\right)$. Then, Birkhoff's ergodic theorem ensures that for $\mu_{n}$-a.e. $x$

$$
\frac{T}{N(x, T)} \rightarrow \int \varrho d \mu_{n} \quad \text { as } T \rightarrow \infty
$$

and, consequently,

$$
\begin{aligned}
\mu_{n}\left(V_{\varepsilon}\right)=\lim _{T \rightarrow \infty} \frac{1}{T} \operatorname{Leb}\left(0 \leq t \leq T: X^{t}(x) \in V_{\varepsilon}\right) & \geq \lim _{T \rightarrow \infty} \frac{N(x, T)}{T} \cdot \frac{1}{N(x, T)} \sum_{j=0}^{N(x, T)-1}(1-\varepsilon)\left(\varrho \cdot \chi_{\Sigma_{\varepsilon}}\right)\left(P^{j}(x)\right) \\
& =(1-\varepsilon) \frac{\int_{\Sigma_{\varepsilon}} \varrho d \mu_{n}}{\int \varrho d \mu_{n}} \geq(1-\varepsilon)\left[1-\frac{C_{\varepsilon}}{\int \varrho d \mu_{n}}\right]
\end{aligned}
$$

for $\mu_{n}$-almost every $x \in \Sigma$. Note that the right hand-side of equation (5.7) tends to $1-\varepsilon$ as $n \rightarrow \infty$. In particular, since $\varepsilon$ was taken arbitrary, any accumulation point $\mu$ of $\left(\mu_{n}\right)_{n}$ satisfies $\mu\left(\overline{V_{\varepsilon}}\right) \geq 1-\varepsilon$ for every $\varepsilon>0$. Hence, we conclude that $\mu_{n} \rightarrow \delta_{\sigma}$ in the weak* topology. This proves the lemma.

Remark 5.3. In the case of singular-hyperbolic attractors, all singularities and periodic orbits are hyperbolic. In particular, for every $T>0$ the set of critical elements formed by singularities and periodic orbits of period smaller than $T$ consists of finitely many disjoint orbits. Since there are countably many critical elements, it is not hard to check that there exists a $C^{0}$-residual subset of $C^{0}(M, \mathbb{R})$ formed by observables $\varphi$ such that

$$
\int \varphi d \mu_{p} \neq \int \varphi d \mu_{q}
$$

for every distinct $p, q \in \operatorname{Crit}(X)$, where $\mu_{p}=\delta_{p}$ if $p$ is a singularity and $\mu_{p}=\frac{1}{\pi(p)} \int_{0}^{\pi(p)} \delta_{X^{s}(p)} d s$ whenever $p$ is a periodic point of period $\pi(p)>0$. An analogous statement also holds for Hölder continuous observables.

5.2. Ergodic optimization for continuous observables. The following lemma gives the starting point to the ergodic optimization of continuous observables, and it ensures that maximizing measures for $C^{0}$-typical observables on singular-hyperbolic attractors (hence for Lorenz attractors) are not supported at singularities.

Lemma 5.4. Let $\Lambda$ be a singular-hyperbolic attractor for $\left(X^{t}\right)_{t}$. The set

$$
O=\left\{\Phi \in C^{0}(N, \mathbb{R}): \int \Phi d \delta_{\sigma}<M\left(\Phi,\left(X^{t}\right)_{t}\right), \forall \sigma \in \operatorname{Sing}(X)\right\}
$$

is a $C^{0}$-open and dense subset of $C^{0}(N, \mathbb{R})$.

Proof. Since the singularities of singular-hyperbolic attractors are hyperbolic, there are finitely many of them, which we denote by $\left\{\sigma_{1}, \sigma_{2}, \ldots, \sigma_{n}\right\}$, and it is clear that $O$ is $C^{0}$-open.

It remains to prove that $O$ is $C^{0}$-dense. Assume that $\Phi \in C^{0}(N, \mathbb{R})$ and that there exists $\sigma_{i} \in \operatorname{Sing}(X)$ so that $\int \Phi d \delta_{\sigma_{i}}=M\left(\Phi,\left(X^{t}\right)_{t}\right)$. We use that for any singularity $\sigma_{i} \in \Lambda$ the probability measure $\delta_{\sigma_{i}}$ is accumulated by invariant and ergodic measures associated to periodic measures $\left(\mu_{p}\right)_{p \in \operatorname{Per}\left(\left(X^{t}\right)_{t}\right)}($ recall Lemma 5.2). In particular,

$$
M\left(\Phi,\left(X^{t}\right)_{t}\right)=\int \Phi d \delta_{\sigma_{i}}=\sup _{p \in \operatorname{Per}\left(\left(X^{t}\right)_{t}\right)} \int \Phi d \mu_{p} .
$$


For any $\varepsilon>0$ let $p \in \operatorname{Per}\left(\left(X^{t}\right)_{t}\right)$ be such that $\int \Phi d \mu_{p}>\int \Phi d \delta_{\sigma_{i}}-\frac{\varepsilon}{2}$ and let $0<\delta=\delta\left(\sigma_{i}, p\right) \ll \varepsilon$ be such that $\min _{t \in[0, \pi(p)]} \operatorname{dist}\left(\sigma_{i}, X^{t}(p)\right) \geq 2 \delta>0$. Performing a $\varepsilon$ - $C^{0}$-small perturbation supported in a $\delta$-neighborhood of $p$ we obtain a $C^{0}$-observable $\Phi_{1}$ so that $\Phi_{1} \equiv \Phi$ on $M \backslash B\left(\sigma_{i}, \delta\right)$ and $\Phi_{1}\left(\sigma_{i}\right)<\Phi\left(\sigma_{i}\right)-\varepsilon$. In particular

$$
\int \Phi_{1} d \delta_{\sigma_{i}}<\int \Phi d \delta_{\sigma_{i}}-\varepsilon<\int \Phi d \mu_{p}-\frac{\varepsilon}{2}=\int \Phi_{1} d \mu_{p}-\frac{\varepsilon}{2} \leq M\left(\Phi_{1}\right)-\frac{\varepsilon}{2},
$$

hence $\delta_{\sigma_{i}}$ is not a $\Phi_{1}$-maximizing measure. Since there are finitely many singularities, after a finite number of $C^{0}$-perturbations with disjoint supports we obtain a $C^{0}$-observable $\Phi_{k}$ that is $\varepsilon$ - $C^{0}$-close to $\Phi_{k}$ for which no Dirac measure at a singularity is a $\Phi_{k}$-maximizing measure. This shows that $\Phi_{k} \in O$ and proves the lemma.

Let $O$ be given by the previous lemma. Using Theorem 2.1 together with Remarks 2.2 and 2.3, we conclude that there exists a $C^{0}$-residual subset $\Re_{1} \subset C^{0}(N, \mathbb{R})$ so that every $\Phi \in \mathcal{R}$ has a unique maximizing measure. Consider the Baire residual subset $\mathcal{R}_{1}:=\Re_{1} \cap O$. By construction, every $\Phi \in \mathcal{R}_{1}$ has a unique maximizing measure $\mu$ (hence ergodic). Item (1) of Theorem B and the following characterization of the support of maximizing measures:

Lemma 5.5. Let $\Lambda$ be a Lorenz attractor. There exists a $C^{0}$-Baire generic subset of $C^{0}(N, \mathbb{R})$ formed by observables which have a unique maximizing measure $\mu$, which is non-atomic and so that $\operatorname{supp} \mu$ contains a singularity.

Proof. Let $\Phi \in \mathcal{R}_{1}$ and let $\mu$ be the unique (ergodic) $\Phi$-maximizing measure. Since $\mathcal{R}_{1} \subset O$ then $\mu$ cannot be the Dirac mass at the singularity. In order to conclude that $\mu$ is non-atomic it is enough to show that the support of $\mu$ contains the singularity. This requires extra information, of independent interest, on the relative maximization at hyperbolic sets as follows.

For any $\varepsilon>0$ let $\mathcal{U}_{\varepsilon}$ denote the $\varepsilon$-neighborhood of $\operatorname{Sing}(X)$. We describe three situations to consider for the shape of the non-increasing function $\varepsilon \rightarrow M_{\mathcal{U}_{\varepsilon}}(\Phi)$ (see Figure 2). In cases a. and b. we have $M_{\mathcal{U}_{\varepsilon}}(\Phi)<M(\Phi)$
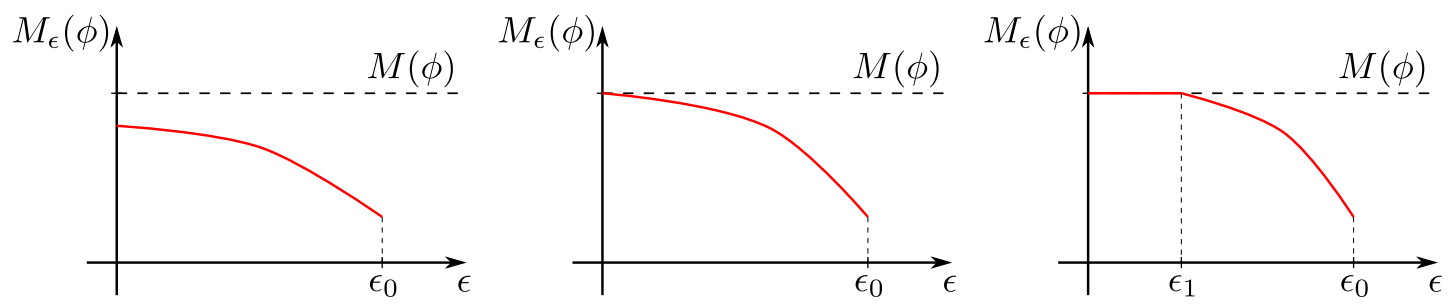

FIGURE 2. Possible shapes for the function $\varepsilon \rightarrow M_{\mathcal{U}_{\varepsilon}}(\Phi)$

for every $\varepsilon>0$. If $\operatorname{supp} \mu \subset \Lambda$ contains no singularity then there exists $\varepsilon_{0}>0$ such that $\operatorname{supp} \mu \cap \mathcal{U}_{\varepsilon_{0}}=\emptyset$, thus supp $\mu \subset \Lambda_{\mathcal{U}_{\varepsilon_{0}}}$ contradicting the fact that $M_{\mathcal{U}_{\varepsilon_{0}}}(\Phi)<M(\Phi)$. Thus proves that supp $\mu \subset \Lambda$ contains a singularity.

We claim that case c. does not occur for $C^{0}$-generic observables. Indeed, let $\Phi \in C^{0}(N, \mathbb{R})$ and $\varepsilon_{1}>0$ be so that $M_{\mathcal{U}_{\varepsilon}}(\Phi)=M\left(\Phi,\left(X^{t}\right)_{t}\right)$ for every $0<\varepsilon \leq \varepsilon_{1}$. Pick $0<\varepsilon_{2}<\varepsilon_{1}$ such that $\Lambda_{\mathcal{U}_{\varepsilon_{2}}} \subsetneq \Lambda_{\mathcal{U}_{\varepsilon_{1}}}$. Using that each map $C^{0}(N, \mathbb{R}) \rightarrow C^{0}\left(\Lambda_{\mathcal{U}_{\varepsilon_{i}}}, \mathbb{R}\right)$ given by $\left.\Phi \mapsto \Phi\right|_{\Lambda_{\mathcal{u}_{i}}}$ is a submersion $(i=1,2)$ and Theorem A we deduce that there exist $C^{0}$-Baire residual subsets $\mathcal{R}_{\varepsilon_{1}}, \mathcal{R}_{\varepsilon_{2}} \subset C^{0}(N, \mathbb{R})$ such that every $\Psi \in \mathcal{R}_{i}$ has a unique $\Psi$-maximizing measure on $\Lambda_{\mathcal{U}_{\varepsilon_{i}}}$, and its support coincides with $\Lambda_{\mathcal{U}_{\varepsilon_{i}}}(i=1,2)$. Therefore, any observable $\Psi$ in the $C^{0}$-Baire residual subset $\mathcal{R}_{1} \cap \mathcal{R}_{\varepsilon_{1}} \cap \mathcal{R}_{\varepsilon_{2}}$ has a unique maximizing measure on $\Lambda, \Lambda_{\mathcal{U}_{\varepsilon_{2}}}$ and $\Lambda_{\mathcal{U}_{\varepsilon_{1}}}$. Since $M \mathcal{U}_{\varepsilon_{1}}(\Phi)=M_{\mathcal{U}_{\varepsilon_{2}}}(\Phi)=M\left(\Phi,\left(X^{t}\right)_{t}\right)$ we conclude that $\mu_{\varepsilon_{2}}=\mu_{\varepsilon_{1}}$, which contradicts the fact that $\Lambda_{\mathcal{U}_{\varepsilon_{2}}} \subsetneq \Lambda_{\mathcal{U}_{\varepsilon_{1}}}$. Thus, $C^{0}$-generic observables do not satisfy case c. This finishes the proof of the lemma. 
5.3. Ergodic optimization for generic Lorenz attractors. In this subsection we will prove Corollary 1. Let $M$ be a 3-dimensional compact boundless Riemannian manifold and let $\mathcal{R} \subset \mathfrak{X}^{1}(M)$ be the $C^{1}$-residual subset given by Proposition 2.11.

Given a vector field $X \in \mathcal{R}$, by item (1) of Theorem B there exists a $C^{0}$-residual subset of observables $\mathcal{R}_{X} \subset C^{0}(N, \mathbb{R})$ such that every $\Phi \in \mathcal{R}_{X}$ has a unique $\Phi$-maximizing measure $\mu$ with respect to $\left(X^{t}\right)_{t}$, whose support contains a singularity and is not atomic. Then, for $\mu$-almost every $x$ there exists $y \in W^{u}(\sigma) \backslash\{\sigma\}$ such that $y \in \omega(x)$. Using continuous dependence on initial conditions and the fact that $\overline{W^{u, \pm}(\sigma)}=\Lambda$ we conclude that $\operatorname{supp} \mu=\Lambda$. By construction the residual subset $\bigcup_{X \in \mathcal{R}}\{X\} \times \mathcal{R}_{X} \subset \mathfrak{X}^{1}(M) \times C^{0}(N, \mathbb{R})$ satisfies the requirements of the corollary.

5.4. Ergodic optimization for Hölder continuous observables. Here we prove item (2) in Theorem B. Using that $C^{\alpha}(N, \mathbb{R}) \subset C^{0}(N, \mathbb{R})$ is a $C^{0}$-dense subspace, Theorem 2.1 and Remarks 2.2 and 2.3, we conclude that there exists a $C^{\alpha}$-residual subset $\Re_{2} \subset C^{\alpha}(N, \mathbb{R})$ so that every $\Phi \in \mathfrak{R}_{2}$ has a unique $\Phi$-maximizing measure. Given $\Phi \in \mathfrak{R}_{2}$, if $\mu$ denotes the unique $\Phi$-maximizing measure then there are three cases to consider:

(i) If $M(\Phi)=\Phi(\sigma)$ then $\mu=\delta_{\sigma}$ is the Dirac measure at the singularity;

(ii) If there exists $\varepsilon_{0}>0$ such that $M_{\mathcal{U}_{\varepsilon}}(\Phi)=M(\Phi)$ for every $0<\varepsilon \leq \varepsilon_{0}$, since the restriction map $C^{\alpha}(N, \mathbb{R}) \rightarrow C^{\alpha}\left(\Lambda_{\mathcal{U}_{\varepsilon}}, \mathbb{R}\right)$ is a submersion and generic Hölder observables on hyperbolic sets have maximizing measures supported on periodic orbits we conclude that the unique maximizing measure $\mu$ of a $C^{\alpha}$-Baire generic observable it is supported on a periodic orbit in $\Lambda_{\mathcal{U}_{\varepsilon_{0}}}$; and

(iii) If $M(\Phi)>\Phi(\sigma)$ and $M_{\mathcal{U}_{\varepsilon}}(\Phi)<M(\Phi)$ for every $\varepsilon>0$, the same argument involved in the proof of item (1) in Theorem B ensures that $\mu$ is not atomic and its support contains the singularity.

This finishes the proof of item (2) in Theorem B.

Remark 5.6. We expect that $C^{\alpha}$-generic observables have unique maximizing measures supported at some critical element, similarly to the case of hyperbolic basic sets. This is the case if one assures that item (iii) above holds for observables in a meager subset. This is not immediate as the periodic measures for the restricted maximum $M_{\mathcal{U}_{\varepsilon}}(\Phi)$ could have smaller frequency of visits to neighborhoods of the singularity as $\varepsilon$ tends to zero, but still to accumulate on a invariant measures having the singularity in its support.

Remark 5.7. The case when the unique maximizing measure is supported at a singularity is somewhat rare. In fact, Lemma 5.4 ensures that any $\Phi \in C^{\alpha}(N, \mathbb{R})$ which has a maximizing measure supported at a singularity is $C^{0}$-approximated by $C^{0}$-open sets of observables in $C^{\alpha}(N, \mathbb{R})$ for which Dirac measures at singularities are not maximizing measures. In particular there exists a $C^{\alpha}$-open and $C^{0}$-dense subset of the residual subset $\mathcal{R} \subset C^{\alpha}(N, \mathbb{R})$ formed by observables admitting a unique and periodic (non-singular) maximizing measure.

\section{Final COMmENTS}

The ergodic optimization for hyperbolic and singular-hyperbolic flows is still giving first steps and have very few contributions. Let us describe some of the possible future directions of research in this topic. First, while one expects maximizing measures for typical continuous observables to be fully supported and of zero topological entropy, we could not prove this in full strength in Theorem B, as a limitation of the approximation method. Indeed, as maximizing measures are obtained as weak ${ }^{*}$ limits of maximizing measures with zero entropy and fully supported on the approximating horseshoes, these may have positive entropy. A first interesting question is whether the measure theoretic entropies associated to these sequences of maximizing measures has some semi-continuity. Since singular-hyperbolic attractors fail to satisfy the specification property [41], one cannot expect to use the methods in [34]. A second question that arises naturally is related to the proof of Corollary 1 
and the intrinsic nature of singular-hyperbolic attractors: does every compact, nontrivial and transitive subset of a singular-hyperbolic attractor coincide with the attractor itself?

An alternative method to describe the ergodic optimization for three-dimensional singular-hyperbolic attractors (including the Lorenz attractor) could follow from the construction of calibrated sub-actions, following the approach of Lopes and Thieullen [29] in the case of Anosov flows. Indeed, since singular-hyperbolic attractors admit suitable cross-sections and can be modeled by suspension flows over maps with countable Markov partitions (see e.g. [2]), it sounds reasonable that the construction of sub-actions for Young towers in [15] may be pushed to the context of suspension flows of maps modeled by Young towers and ultimately to the realm of singular-hyperbolic attractors. The ergodic optimization for Lorenz attractors and Hölder potentials seems not immediate from this method. Indeed, the $C^{0}$-topology is crucial in order to perturb observables in a small open neighborhood of the singularities (recall e.g. Lemma 5.4).

A further interesting question, inspired by [17], concerns the case of maximizing measures for the Lyapunov exponents. In the case of three-dimensional hyperbolic flows, this corresponds to the description of the maximizing measures with respect to the observable $-\log \left|\operatorname{det} D X^{t}\right| E^{u} \mid$. In this case the vector field and the observable are coupled, which demands subtle perturbations of the underlying dynamics.

Finally, it seems challenging to describe the ergodic optimization of continuous flows (or Lipschitz vector fields) when the observable is fixed. Such a problem was addressed in [1] in the discrete-time context, but an extension to the context of continuous flows seems to face fundamental difficulties raised by the lack of perturbation methods for flows with low regularity.

Acknowledgements. This work was part of the first author's PhD thesis at UFBA, and it was partially supported by CNPq-Brazil and CAPES-Brazil. The third author was partially supported by CNPq-Brazil and by FCT-Portugal. The authors are indebted to V. Araújo, F. Pedreira, V. Pinheiro and X. Tian for useful comments and calling their attention to $[22,32,35]$.

\section{REFERENCES}

[1] S. Addas-Zanata and F. Tal, Support of maximizing measures for typical $C^{0}$ dynamics on compact manifolds, Discrete Cont. Dyn. Syst., 26 (2010) 795-804.

[2] V. Araújo and M. J. Pacífico, Three-dimensional flows, Springer Science \& Business Media, volume 53, 2010.

[3] V. Araújo and P. Varandas. Robust exponential decay of correlations for singular-flows. Comm. Math. Phys., 311:215-246, 2012.

[4] A. T. Baraviera, R. Leplaideur and A. Lopes, Ergodic optimization, zero temperature limits and the max-plus algebra, IMPA, 2013, $29^{\circ}$ Coloquio Brasileiro de Matematica.

[5] A. T. Baraviera, R. Leplaideur and A. O. Lopes. Selection of ground states in the zero temperature limit for a one-parameter family of potentials. SIAM J. Appl. Dyn. Syst. 11 (2012), 243-260.

[6] L. Barreira, Dimension theory of hyperbolic flows, Springer-Verlag, 2013.

[7] T. Bomfim and P. Varandas, The gluing orbit property, uniform hyperbolicity and large deviation principles for semiflows, J. Diff. Equations, 267 (2019) 228-266.

[8] T. Bomfim, M. J. Torres and P. Varandas, Topological features of flows with the reparametrized gluing orbit property, J. Diff. Equations, 262:8 (2017) 4292-4313.

[9] R. Bissacot and E. Garibaldi. Weak KAM methods and ergodic optimal problems for countable Markov shifts. Bull. Braz. Math. Soc. (N.S.) 41 (2010), 321-338.

[10] J. Bochi and Y. Zhang. Ergodic optimization of prevalent super-continuous functions. Int. Math. Res. Notices IMRN 19 (2016), 5988-6017.

[11] T. Bousch, Le poisson n'a pas d'arètes, Ann. Inst. H. Poincaré Probab. Statist., 36:4 (2000) 489-508.

[12] R. Bowen. Symbolic dynamics for hyperbolic flows. Amer. J. Math., 95(1973) 429-460.

[13] R. Bowen, Equilibrium states and the ergodic theory of Anosov diffeomorphisms, Springer Lecture Notes in Math., vol. $470,1975$.

[14] R. Bowen and P. Walters, Expansive one-parameter flows, J. Diff. Equations, 12:1 (1972) 180-193.

[15] S. Branton. Sub-actions for Young towers. Discrete Cont. Dyn. Syst. 22 (2008), 541-556.

[16] J. Brémont. Entropy and maximizing measures of generic continuous functions. C. R. Math. Acad. Sci. Sér. I 346 (2008), $199-201$. 
[17] G. Contreras, A. Lopes, and $\mathrm{Ph}$ Thieullen, Lyapunov minimizing measures for expanding maps of the circle, Ergodic Theory and Dynamical Systems, 21:5 (2001) 1379-1409.

[18] G. Contreras, Ground states are generically a periodic orbit, Invent. Math., 205:2 (2016) 383-412.

[19] G. Contreras, Generic Mañé sets, https://arxiv.org/pdf/1410.7141.pdf

[20] A. Fathi, Théorème KAM faible et théorie de Mather sur les systèmes lagrangiens, C. R. Acad. Sci. Paris Sér. I Math., $324: 9$ (1997) 1043-1046.

[21] E. Garibaldi, A. Lopes and Ph. Thieullen, On calibrated and separating sub-actions, Bull. Braz. Math. Soc. (N.S.), 40:4 (2009) 577-602.

[22] B. Han and X. Wen, A shadowing lemma for quasi-hyperbolic strings of flows, J. Diff. Equations 264 (2018) 1-29.

[23] M. Hirsch, J. Palis, C. Pugh and M. Shub, Neighborhoods of hyperbolic sets, Invent. Math., 9:2 (1970) 121-134.

[24] W. Huang, Z. Lian, X. Ma, L. Xu and Y. Zhang Ergodic optimization theory for a class of typical maps, Preprint ArXiv:1904.01915

[25] W. Huang, Z. Lian, X. Ma, L. Xu and Y. Zhang Ergodic optimization theory for non-degenerate Axiom A flows, Preprint Researchgate 2019

[26] O. Jenkinson, Ergodic optimization, Discrete Cont. Dyn. Syst., 15:1 (2006) 197.

[27] O. Jenkinson, Survey: Ergodic optimization in dynamical systems, Ergod. Th. Dynam. Sys. (2018) doi:10.1017/etds.2017.142

[28] A. Katok, B. Hasselblatt. Introduction to the modern theory of dynamical systems. Encyclopedia of Mathematics and its Aplications, 1997.

[29] A. Lopes and Ph. Thieullen, Sub-actions for Anosov flows, Ergodic Theory and Dynamical Systems, 25:2 (2005) $605-628$.

[30] A. Lopes, V. Rosas and R. Ruggiero Cohomology and subcohomology for expansive geodesic flows, Discrete Cont. Dyn. Syst., 17:2 (2007) 403-422.

[31] R. Mañé, Lagrangian flows: the dynamics of globally minimizing orbits, Bull. Braz. Math. Soc., 28:2 (1997) 141-153.

[32] C. Morales and M. J. Pacífico, Mixing attractors for 3-flows, Nonlinearity 14 (2001) 359-378.

[33] I. D. Morris. A sufficient condition for the subordination principle in ergodic optimization. Bull. Lond. Math. Soc. 39 (2007), 214-220.

[34] I. D. Morris, Ergodic optimization for generic continuous functions, Discrete Cont. Dyn. Sys, 27 (2010) 383-388.

[35] F. Pedreira, Genericity of expanding Lorenz maps with slow recurrence, PhD Thesis - UFBA, to appear (PhD Advisor: Vilton Pinheiro)

[36] M. Pollicott and R. Sharp, Livsic theorems, maximizing measures and the stable norm, Dyn. Syst., 19 (2004), 75-88.

[37] A. Quas and J. Siefken, Ergodic optimization of super-continuous functions on shift spaces, Ergod. Th. Dynam. Sys., 32:6 (2012) 2071-2082.

[38] M. Ratner, Markov partitions for Anosov flows on n-dimensional manifolds, Israel J. Math., 15:1 (1973) 92-114.

[39] M. Shinoda, Uncountably many maximizing measures for a dense subset of continuous functions, Nonlinearity 31 (2018) 21922200.

[40] W. Sun, X. Tian and E. Vargas, Non-uniformly hyperbolic flows and shadowing, J. Diff. Equations 261 (2016) 218-235

[41] N. Sumi, P. Varandas and K. Yamamoto, Specification and partial hyperbolicity for flows, Dynam. Sys., 30:4, (2015) $501-524$.

[42] K. Sigmund, Generic properties of invariant measures for Axiom A diffeomorphisms, Invent. Math. 11 (1970) 99-109.

[43] L.-S. Young, On the prevalence of horseshoes, Trans. Amer. Math. Soc., 263:1 (1981), 75-88.

[44] G. Yuan and B. Hunt, Optimal orbits of hyperbolic systems, Nonlinearity, 12: (1999) 1207-1224.

Marcus Morro, Departamento de Matemática e Estatística, Universidade Federal da Bahia, Av. Ademar de Barros s/n, $40170-110$ SALVADOR, Brazil

E-mail address: marcusmorro@gmail.com

Roberto Sant'Anna, Departamento de Matemática e Estatística, Universidade Federal da Bahia, Av. Ademar de Barros s/N, 40170-110 SALVADOR, BRAZIL

E-mail address: rsantanna@ufba.br

Paulo Varandas, Departamento de Matemática e Estatística, Universidade Federal da Bahia, Av. Ademar de Barros s/N, 40170110 Salvador, Brazil \& CMUP, University of Porto, Rua do Campo Alegre 687, 4169-007 Porto, Portugal

E-mail address: pcvarand@gmail.com

URL: https://sites.google.com/view/paulovarandas/ 